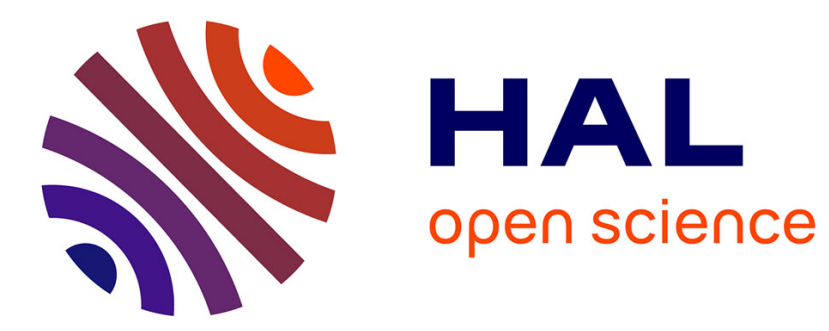

\title{
Neutron transport in anisotropic random media
}

Alessandro Marinosci, Coline Larmier, Andrea Zoia

\section{To cite this version:}

Alessandro Marinosci, Coline Larmier, Andrea Zoia. Neutron transport in anisotropic random media. Annals of Nuclear Energy, 2018, 118, pp.406-413. 10.1016/j.anucene.2018.04.031 . cea-02339792

\section{HAL Id: cea-02339792 https://hal-cea.archives-ouvertes.fr/cea-02339792}

Submitted on 5 Nov 2019

HAL is a multi-disciplinary open access archive for the deposit and dissemination of scientific research documents, whether they are published or not. The documents may come from teaching and research institutions in France or abroad, or from public or private research centers.
L'archive ouverte pluridisciplinaire HAL, est destinée au dépôt et à la diffusion de documents scientifiques de niveau recherche, publiés ou non, émanant des établissements d'enseignement et de recherche français ou étrangers, des laboratoires publics ou privés. 


\title{
Neutron transport in anisotropic random media
}

\author{
Coline Larmier ${ }^{\mathrm{a}}$, Alessandro Marinosci ${ }^{\mathrm{b}}$, Andrea Zoia ${ }^{\mathrm{a}, *}$ \\ ${ }^{a} D E N$-Service d'études des réacteurs et de mathématiques appliquées (SERMA), CEA, \\ Université Paris-Saclay, F-91191, Gif-sur-Yvette, France \\ ${ }^{b}$ Politecnico di Torino, Corso Duca degli Abruzzi 24, 10129, Torino, Italy.
}

\begin{abstract}
Assessing the impact of random media for eigenvalue problems plays a central role in nuclear reactor physics and criticality safety. In a recent work (Larmier et al., 2018a), we have applied a probabilistic model based on stochastic tessellations in order to describe fuel degradation following severe accidents with partial melting and re-arrangement of the resulting debris. The distribution of the multiplication factor and of the kinetics parameters as a function of the mixing statistics model and of the typical correlation length of the tessellation were examined in detail for a benchmark configuration consisting in a fuel assembly with UOX or MOX fuel pins. In this paper, we extend our previous findings by including in the stochastic tessellation model the effects of anisotropy that might result from gravity and material stratification: for this purpose, we adopt the broad class of anisotropic Poisson geometries. We examine the evolution of the statistical properties of the tessellations, including the volume, surface and chord length of the cells for various anisotropy laws, and compare them to the case of isotropic Poisson geometries. Then, we discuss the behaviour of the key observables of interest for eigenvalue problems in anisotropic tessellations by revisiting the fuel assembly benchmark calculations proposed in (Larmier et al., 2018a). The effects of anisotropic random media on the multiplication factor and on the kinetics parameters will be carefully examined.
\end{abstract}

Keywords: Random media, Markov tessellations, Anisotropy, Monte Carlo, TRIPOLI-4 ${ }^{\circledR}$, Kinetics parameters.

\footnotetext{
*Corresponding author

Email address: andrea.zoia@cea.fr (Andrea Zoia)
} 


\section{Introduction}

Random media of interest in reactor physics typically belong to two families: stochastic inclusions of fissile chunks within a background matrix (Murata et al., 1996; Liang et al., 2013; Brown and Martin, 2004) and stochastic tessella5 tions composed of a collection of fissile and non-fissile volumes obeying a given mixing statistics (Pomraning, 1991a), such as those resulting from fuel degradation in Three Mile Island unit 2 (Broughton et al., 1989; Hagen and Hofmann, 1987; Hofmann, 1999) and at the Fukushima Daiichi power plant (Tonoike et al., 2013; Gunji et al., 2017). Other applications of random media for criticality safety concern for instance the analysis of the impact of poison grains for neutron absorbers (Doub, 1961) or Pu grains in MOX fuels (Yamamoto, 2010), and safety margins evaluation (Pomraning, 1999; Williams, 2000; Williams and Larsen, 2001; Williams, 2013), especially for waste storage (Williams, 2003).

Two distinct strategies can be adopted in order to describe neutron multiplication in random media (Pomraning, 1991a), namely, quenched disorder and annealed disorder. The goal of the annealed disorder approach is to develop effective equations for the ensemble-averaged observables, such as the celebrated Levermore-Pomraning model (Pomraning, 1991a). When particle transport is solved by Monte Carlo simulation, the annealed disorder approach is implemented by introducing disorder-averaged neutron displacement laws that are supposed to 'mimic' the effects of the spatial heterogeneities on neutron trajectories: this is for instance the case of Chord Length Sampling (CLS) algorithm, inspired by the Levermore-Pomraning equations (Zimmerman, 1990; Zimmerman and Adams, 1991; Donovan and Danon, 2003; Donovan et al., 2003). By construction, spatial correlations are neglected by these algorithms. Generalizations of CLS including partial memory effects and spatial correlations have been also proposed (Zimmerman and Adams, 1991; Donovan and Danon, 2003; Donovan et al., 2003; Larmier et al., 2018c). In order to assess the accuracy of such approximate methods, reference solutions are mandatory (Levermore et so al., 1986; Adams et al., 1989; Malvagi et al., 1992; Su and Pomraning, 1995; Zuchuat et al., 1994; Larsen and Vasques, 2011; Brantley, 2011; Donovan and Danon, 2003; Donovan et al., 2003; Brantley and Palmer, 2009; Brantley, 2009; Larmier et al., 2018b).

In the quenched disorder approach, the random spatial configurations (with associated material compositions) are first defined based on a probabilistic model. The Boltzmann eigenvalue equation is then solved for each configuration, and the statistical moments of the multiplication factor and of the kinetics parameters are obtained by taking the ensemble averages with respect to the realiza- 
tions (Pomraning, 1991a,b, 1999). The quenched disorder approach leads to reference solutions, because the effects of disorder-induced spatial correlations on neutron trajectories are correctly preserved. Analytical results for the ensemble averages demand huge theoretical efforts (Pomraning, 1999; Williams, 2000, 2003; Williams and Larsen, 2001; Williams, 2004): advances have been made possible by resorting to perturbation theory, but several simplifications are needed (Pomraning, 1999; Williams and Larsen, 2001; Williams, 2004). Considerable progress can be nonetheless achieved by using Monte Carlo methods in order to generate realizations taken from the sought distribution and then using a transport code to solve the eigenvalue problem for each sampled configuration. In the context of eigenvalue problems, intensive research efforts have been devoted so far to the class of stochastic inclusions (Murata et al., 1996; Liang et al., 2013; Brown and Martin, 2004; Liang and Ji, 2011; Grieshiemer et al., 2010; $\mathrm{Ji}$ and Martin., 2013). Eigenvalue problems for stochastic tessellations have comparatively received less attention, and have been mostly confined to onedimensional systems (Pomraning, 1999; Williams and Larsen, 2001; Williams, 2004).

In order to overcome some of these limitations, in a recent work we have adopted three-dimensional stochastic tessellations as a idealized model to investigate neutron kinetics following random fuel degradation (Larmier et al., 2018a). For this purpose, we have introduced a series of benchmark configurations consisting of a $17 \times 17$ UOX or MOX assembly, where a portion of the assembly was replaced by a Poisson, Voronoi or Box tessellation with fuel, cladding and moderator obeying ternary mixing statistics (Pomraning, 1991a). For each realization, criticality calculations were performed by using the Monte Carlo transport code TRIPOLI-4 ${ }^{\circledR}$ developed at CEA (Brun et al., 2015). We assessed the behaviour of the key safety parameters, including the multiplication factor $k_{\text {eff }}$, the effective delayed neutron fraction $\beta_{\text {eff }}$ and the effective neutron generation time $\Lambda_{\text {eff }}$, as a function of the tessellation law and of the size of the material chunks.

For real life applications, fuel degradation will generally give rise to anisotropic re-arrangements, possibly due to gravity and other material stratification phenomena (Hagen and Hofmann, 1987; Hofmann, 1999). To the best of our knowledge, neutron transport in anisotropic random media has been only considered in relation to the Levermore-Pomraning model (Pomraning, 1992). For the sake of simplicity, the stochastic tessellations investigated in Larmier et al. (2018a) 75 were isotropic (Poisson and Voronoi) or quasi-isotropic (Box). In this work, we will extend our previous results concerning reference solutions for eigenvalue 
problems in random media by relaxing the isotropy hypothesis, with a two-fold aim: first, we will illustrate how the statistical features of the cells composing the tessellations (the typical volume, surface, number of faces, the chord length distribution, and so on) evolve as a function of the anisotropy law; then, we will show how the geometrical properties of the underlying tessellations affect the key safety parameters for eigenvalue problems in stochastic media.

This paper is organized as follows: in Sec. 2 we will briefly introduce the class of $d$-dimensional anisotropic Poisson stochastic tessellations and detail their main statistical properties. In Sec. 3 we will numerically verify the behaviour of three-dimensional anisotropic Poisson tessellations by Monte Carlo methods for different anisotropy laws. An application to the analysis of neutron multiplication in random media will be illustrated in Sec. 4, where we will revisit the tessellation-based fuel assembly model introduced in (Larmier et al., 2018a). Then, simulation results for the multiplication factor and the kinetics parameters will be analysed in Sec. 5. Conclusions will be finally drawn in Sec. 6. Additional technical details will be provided in Appendix A.

\section{Anisotropic Poisson tessellations}

Consider a disjoint aggregate $\left\{X_{i}\right\}, i=1,2, \cdots$ of bounded domains in the euclidean space $\mathbb{R}^{d}$, with $X_{i} \cap X_{j}=\emptyset$ for $i \neq j$. The aggregate is space-filling in $\mathbb{R}^{d}$ if the union of the domains $X_{i}$ yields the entire space $\mathbb{R}^{d}$. When the bounded domains are convex and space-filling, the interfaces between domain pairs must be hyperplanes, and the domains $X_{i}$ must be convex polyhedra. In this case, the aggregate $\left\{X_{i}\right\}$ is a tessellation of $\mathbb{R}^{d}$, and the domains $X_{i}$ are the cells of the tessellation. Stochastic tessellations are a class of probabilistic models where a given $d$-dimensional domain is decomposed into random convex polyhedral cells by generating a collection of random hyperplanes in $\mathbb{R}^{d}$ (Miles, 1970; Santalo, 1976; Torquato, 2013; Schneider and Weil, 2008; Chiu et al., 2013). Applications encompass material science (Gilbert, 1962; Meijering, 1953) and stereology (Serra, 1982; Santalo, 1976): a comprehensive review can be found, e.g., in (Santalo, 1976; Schneider and Weil, 2008; Torquato, 2013). Poisson geometries are a prominent example of stochastic tessellations: a domain included in a $d$-dimensional space is partitioned by randomly generated $(d-1)$-dimensional hyperplanes drawn from an underlying Poisson process (Miles, 1970; Santalo, 1976; Schneider and Weil, 2008). Poisson tessellations represent an idealized mathematical model for disordered media: they demand little information content, their correlation function being exponential, as detailed later (Pomraning, 1991a; Torquato, 2013). 
The key properties of Poisson geometries, such as the volume of a typical cell, its surface, the number of faces, and so on, are random variables, whose exact distributions are in most cases unfortunately not known (Schneider and Weil, 2008). Nevertheless, analytical expressions have been established for a few loworder moments of the observables, in the limit case of domains having an infinite extension (Santalo, 1976; Schneider and Weil, 2008; Miles, 1970). The case of isotropic Poisson tessellations was first addressed by the pioneering work of Goudsmit for $d=2$ (Goudsmit, 1945) and later thoroughly explored by Miles first for $d=2$ and later for higher dimensions in a series of ground-breaking works (Miles, 1964, 1970, 1972a). Not surprisingly, the derivation of exact results for anisotropic tessellations is even more demanding: the case $d=2$ was investigated by Miles (Miles, 1964), whereas formulas for higher dimensions were found much later and are still a subject of active research (Schneider and Weil, 2008; Schneider, 2013, 2016).

A method for the explicit construction of $d$-dimensional homogeneous and isotropic Poisson tessellations restricted to finite domains has been proposed (Serra, 1982; Ambos and Mikhailov, 2011). In the following we will detail the algorithm for the construction of Poisson tessellations based on Monte Carlo methods and show that anisotropy can be rather straightforwardly taken into account. In view of using these stochastic tessellations to derive reference solutions for particle transport in random media, we have implemented this algorithm into a computer code that can generate an ensemble of realizations. For nummerical verification of the construction algorithms, the code estimates the moments and the distributions for the sought observables, which allows numerically investigating the statistical features of the resulting tessellations. The main theoretical results concerning anisotropic Poisson tessellations will be recalled and compared to our simulation findings, similarly as previously done for the case of isotropic Poisson tessellations (Larmier et al., 2016).

\subsection{Construction of Poisson tessellations}

We illustrate a method for the construction of anisotropic Poisson geometries restricted to a $d$-dimensional box. We start by sampling a random number $N$ of hyperplanes from a Poisson distribution of intensity $\alpha_{d} \rho R$, where $R$ is the radius of the $d$-sphere circumscribed to the box and $\rho$ is the so-called density of the tessellation, carrying the units of an inverse length. The dimension-dependent constant $\alpha_{d}$ reads

$$
\alpha_{d}=\frac{\Omega_{d}}{\kappa_{d-1}}=\frac{d \kappa_{d}}{\kappa_{d-1}}
$$


where

$$
\Omega_{d}=\frac{2 \pi^{d / 2}}{\Gamma\left(\frac{d}{2}\right)}
$$

150

is the surface of the unit $d$-sphere and

$$
\kappa_{d}=\frac{\pi^{d / 2}}{\Gamma\left(1+\frac{d}{2}\right)}
$$

is the volume of the unit $d$-sphere. This normalization of the tessellation density is such that $\rho$ yields the mean number of $(d-1)$-hyperplanes intersected by an arbitrary segment of unit length (Santalo, 1976). Then, we generate the hyperplanes $K$ that will cut the box. We choose a parameter $r$ uniformly in the interval $[-R, R]$ and additionally sample a unit vector $\mathbf{n}$ from a density $H(\mathbf{n})$ with support in $\Omega_{d}^{+}=\Omega_{d} / 2$, i.e., the half-surface of the unit $d$-sphere. Denoting by $\mathbf{M}$ the point such that $\mathbf{O M}=r \mathbf{n}$, the random plane $K$ will pass trough $\mathbf{M}$ and have normal vector $\mathbf{n}$ (for an illustration in $d=3$, see the scheme in Fig. 1). The polyhedra defined by the intersections of such random planes are convex. By construction, this hyperplane does intersect the circumscribed $d$-sphere of radius $R$ but not necessarily the box. The procedure is iterated until $N$ random hyperplanes have been generated, which yields homogeneous but (generally) non-isotropic Poisson tessellations (Schneider and Weil, 2008). Anisotropy affects the construction of the tessellations through the distribution $H(\mathbf{n})$ : isotropic Poisson tessellations are obtained as a special case when setting $H(\mathbf{n})=U(\mathbf{n})$, i.e., the uniform angular distribution over $\Omega_{d}^{+}$(for further details on the isotropic case, see (Larmier et al., 2016)). Due to geometrical reasons, it is only possible to include anisotropy effects for $d \geq 2$. A few examples of realizations corresponding to various angular laws $H(\mathbf{n})$ are illustrated in Fig. 2. The density $H(\mathbf{n})$ might admit a finite mass concentrated on a given direction $\mathbf{n}_{0}$ (a so-called atom), or more generally on a collection of discrete directions $\mathbf{n}_{i}$, with Dirac delta distributions: the special case where the discrete directions $\mathbf{n}_{i}, i=1, \cdots, d$, are chosen parallel to the Cartesian axes in dimension $d$ gives rise to the so-called Poisson-Box tessellations (Miles, 1964, 1972a).

\subsection{Statistical properties of polyhedral cells}

Knowledge of the statistical properties of the polyhedral cells composing the stochastic tessellations can be helpful in understanding the particle streaming through the geometry. The quantities of interest in the framework of particle transport are the volume $V_{d}$ of a typical cell in dimension $d$, which characterizes the spatial scale of the random medium, and the surface $S_{d}$ and the number of 
faces $f_{d}$ of a cell, which are related to the connectivity of the medium. The inradius $r_{\mathrm{in}, d}$, i.e., the radius of the largest sphere that can be contained in a (convex) polyhedron, and the outradius $r_{\text {out }, d}$, i.e., the radius of the smallest sphere enclosing a (convex) polyhedron, can be also useful so as to characterize the aspect ratio of the cells (for $d=1$, the outradius coincides with the inradius).

\subsubsection{Isotropic tessellations}

For the special case of isotropic Poisson tessellations, the following results have been established for infinite domains (Santalo, 1976; Schneider and Weil, 2008). The inradius $r_{\mathrm{in}, d}$ has an exponential distribution of parameter $\alpha_{d} \rho$, and

$$
\left\langle r_{\mathrm{in}, d}\right\rangle=\frac{1}{\alpha_{d} \rho} .
$$

For the outradius $r_{\text {out }, d}$, the distribution is not known, and to the best of our knowledge the moments are now known, either. For the volume $V_{d}$, we have the moments

$$
\left\langle V_{d}\right\rangle=\frac{1}{\kappa_{d}}\left(\frac{2}{\rho}\right)^{d}
$$

and

$$
\left\langle V_{d}^{2}\right\rangle=\frac{d ! \kappa_{d}^{2}}{2^{d}}\left\langle V_{d}\right\rangle^{2}
$$

The second moment allows computing the coefficient of variation, namely,

$$
\mathrm{cv}=\sqrt{\frac{\left\langle V_{d}^{2}\right\rangle-\left\langle V_{d}\right\rangle^{2}}{\left\langle V_{d}\right\rangle^{2}}},
$$

which conveys information on the spatial shape of a typical cell (Miles, 1972a). For isotropic Poisson tessellations, from Eq. (6) we obtain

$$
\mathrm{cv}=\sqrt{\frac{d ! \kappa_{d}^{2}}{2^{d}}-1 .}
$$

Furthermore, an elegant recursive formula is known relating the correlations between the surface $S_{d}$ and the volume to the higher moments of the volume, namely,

$$
m\left\langle V_{d}^{m-1} S_{d}\right\rangle=\alpha_{d} \rho\left\langle V_{d}^{m}\right\rangle,
$$

for $m \geq 1$ (Miles, 1972a). In particular, for $m=1$ this yields the first moment of $S_{d}$, i.e,

$$
\left\langle S_{d}\right\rangle=\underset{7}{\alpha} \alpha_{d} \rho\left\langle V_{d}\right\rangle
$$


and for $m=2$ the correlations

$$
\left\langle V_{d} S_{d}\right\rangle=\frac{\alpha_{d} \rho}{2}\left\langle V_{d}^{2}\right\rangle
$$

Finally, for the number of faces $f_{d}$ we have the average

$$
\left\langle f_{d}\right\rangle=2 d,
$$

205 which does not depend on $\rho$ and is a purely combinatorial result.

For isotropic Poisson tessellations, many other results are known for the loworder moments and the correlations of $V_{d}, S_{d}$ and $f_{d}$ (Miles, 1964, 1972a; Schneider, 2016), which will not be reported here. For an exhaustive review, the reader is referred to e.g., (Schneider and Weil, 2008).

\subsubsection{Anisotropic tessellations}

As expected, the general case of anisotropic Poisson tessellations is much more involved and the obtained formulas generally depend on the angular distribution $H(\mathbf{n})$. For the polyhedral quantities discussed earlier, the following results have been established for infinite domains (Schneider and Weil, 2008). The distribution of the inradius $r_{\mathrm{in}, d}$ is not affected by the anisotropy of the tessellation, and has again an exponential distribution of parameter $\alpha_{d} \rho$. For the outradius $r_{\text {out }, d}$, no theoretical result is known, to the best of our knowledge. For the volume $V_{d}$ the first moment obeys

$$
\left\langle V_{d}\right\rangle=\frac{d !}{\zeta_{d}}\left(\frac{2}{\alpha_{d} \rho}\right)^{d}
$$

where the constant $\zeta_{d}$ depends on the angular distribution $H(\mathbf{n})$ and is defined as

$$
\zeta_{d}=\int_{\Omega_{d}^{+}} \cdots \int_{\Omega_{d}^{+}}\left[\mathbf{n}_{1}, \cdots, \mathbf{n}_{d}\right] d H\left(\mathbf{n}_{1}\right) \cdots d H\left(\mathbf{n}_{d}\right),
$$

the integrals being extended over the half-surface of the unit $d$-sphere. Here the quantity $\left[\mathbf{n}_{1}, \cdots, \mathbf{n}_{d}\right]$ denotes the $d$-space determinant ${ }^{1}$ of the unit vectors $\mathbf{n}_{1}, \cdots, \mathbf{n}_{d}$ : for $d=2$, e.g., it represents the area of the parallelogram spanned by $\mathbf{n}_{1}$ and $\mathbf{n}_{2}$; for $d=3$, it represents the volume of the parallelepiped spanned by $\mathbf{n}_{1}, \mathbf{n}_{2}$ and $\mathbf{n}_{3}$ (Schneider and Weil, 2008). As a particular case, for isotropic tessellations $H(\mathbf{n})=U(\mathbf{n})$, the integrals in Eq. (14) yield then

$$
\zeta_{d}^{\text {iso }}=\frac{d ! \kappa_{d}}{\alpha_{d}^{d}}
$$

\footnotetext{
${ }^{1}$ If the determinant is negative, we take the absolute value.
} 
and from Eq. (13) we recover Eq. (5) for the average volume. The second moment of the volume $V_{d}$ reads

$$
\left\langle V_{d}^{2}\right\rangle=\zeta_{d} \eta_{d} \kappa_{d}\left\langle V_{d}\right\rangle^{2},
$$

where the constant $\eta_{d}$ also depends on the angular distribution $H(\mathbf{n})$ and is defined as

$$
\eta_{d}=\int_{\Omega_{d}^{+}} d U(\mathbf{v})\left(\int_{\Omega_{d}^{+}}|\mathbf{n} \cdot \mathbf{v}| d H(\mathbf{n})\right)^{-d},
$$

the symbol $|\mathbf{x} \cdot \mathbf{y}|$ denoting the absolute value of the scalar product between $\mathbf{x}$ and $\mathbf{y}$ (Schneider and Weil, 2008). From Eq. (6) we obtain the coefficient of variation

$$
\mathrm{cv}=\sqrt{\zeta_{d} \eta_{d} \kappa_{d}-1}
$$

which generalizes Eq. (8) to the case of arbitrary $H(\mathbf{n})$ distributions. For isotropic tessellations, $H(\mathbf{n})=U(\mathbf{n})$ and the integrals in Eq. (17) yield

$$
\eta_{d}^{\text {iso }}=\left(\frac{\alpha_{d}}{2}\right)^{d}
$$

Hence, from Eq. (16) we recover Eq. (6) for the second moment of the volume. For the surface $S_{d}$, the recursive formula given in Eq. (9) has been shown to hold for any $H(\mathbf{n})$ (Schneider and Weil, 2008), so that the first moment of $S_{d}$ is again related to the average volume by Eq. (10) and the correlations between the surface and the volume are again related to the second moment of the volume by Eq. (11). Thus, the effect of $H(\mathbf{n})$ is only indirectly conveyed on $\left\langle S_{d}\right\rangle$ and $\left\langle V_{d} S_{d}\right\rangle$ through the behaviour of $\left\langle V_{d}\right\rangle$ and $\left\langle V_{d}^{2}\right\rangle$, respectively. It is interesting to observe that, although the average volume and surface depend on the angular law $H(\mathbf{n})$ through the function $\zeta_{d}$, their ratio does not, and we get

$$
\frac{\left\langle V_{d}\right\rangle}{\left\langle S_{d}\right\rangle}=\frac{1}{\alpha_{d} \rho} .
$$

245 Finally, for the average number of faces we have again Eq. (12), which does not depend on the angular distribution.

A special case of anisotropic Poisson tessellations corresponds to taking fixed orientations parallel to the orthogonal Cartesian axes in $\mathbb{R}^{d}$. In the simplest form, each direction is taken with equal probability, which yields a quasi-isotropic stochastic geometry (Miles, 1972a). For these so-called Poisson-Box tessellations, the functions $\zeta_{d}$ and $\eta_{d}$ can be computed exactly from Eqs. (14) and (17), respectively, and yield

$$
\zeta_{d}^{\text {box }}=\frac{d !}{d^{d}}
$$


and

$$
\eta_{d}^{\mathrm{box}}=\frac{(2 d)^{d}}{d ! \kappa_{d}}
$$

\subsubsection{Inequalities for anisotropic tessellations}

It is interesting to remark that the inradius $r_{\mathrm{in}, d}$ and the number of faces $f_{d}$ of Poisson tessellations are actually invariant under the choice of the anisotropy law $H(\mathbf{n})$. On the contrary, the volume $V_{d}$ and the surface $S_{d}$ depend on $H(\mathbf{n})$. It can be shown that for any sufficiently well-behaved $H(\mathbf{n})$ the quantity $\zeta_{d}$ satisfies

$$
\zeta_{d} \leq \frac{d ! \kappa_{d}}{\alpha_{d}^{d}}=\zeta_{d}^{\text {iso }}
$$

which from Eq. (13) implies that the average volume attains a minimum for the case of isotropic Poisson tessellations (Mecke, 1995; Schneider and Weil, 2008), namely,

$$
\left\langle V_{d}\right\rangle=\frac{d !}{\zeta_{d}}\left(\frac{2}{\alpha_{d} \rho}\right)^{d} \geq \frac{1}{\kappa_{d}}\left(\frac{2}{\rho}\right)^{d}=\left\langle V_{d}\right\rangle^{\text {iso }} .
$$

More generally, it can be proven that

$$
\left\langle V_{d}^{m}\right\rangle \geq\left\langle V_{d}^{m}\right\rangle^{\text {iso }}
$$

for the moments of any order $m \geq 1$ (Mecke, 1995), whence in particular

$$
\left\langle V_{d}^{2}\right\rangle=\zeta_{d} \eta_{d} \kappa_{d}\left\langle V_{d}\right\rangle^{2} \geq \frac{d ! \kappa_{d}^{2}}{2^{d}}\left\langle V_{d}\right\rangle^{2}=\left\langle V_{d}^{2}\right\rangle^{\text {iso }}
$$

and $\mathrm{cv} \geq \mathrm{cv}^{\text {iso }}$. For $S_{d}$, Eq. (10) implies that the average surface attains also a minimum for the case of isotropic Poisson tessellations, namely,

$$
\left\langle S_{d}\right\rangle \geq\left\langle S_{d}\right\rangle^{\text {iso }} \text {. }
$$

From Eqs. (26) and (11), we have an analogous inequality for the correlations, namely,

$$
\left\langle V_{d} S_{d}\right\rangle \geq\left\langle V_{d} S_{d}\right\rangle^{\text {iso }}
$$

Finally, numerical investigations show that the average outradius $\left\langle r_{\text {out }, d}\right\rangle$ depends on $H(\mathbf{n})$, contrary to the inradius $\left\langle r_{\mathrm{in}, d}\right\rangle$. 
depending on the anisotropy law $H(\mathbf{n})$. Conversely, the line will be cut by the hyperplanes into segments whose lengths $\ell$ obey the exponential distribution

$$
\mathscr{P}(\ell \mid \mathbf{v})=\rho(\mathbf{v}) e^{-\ell \rho(\mathbf{v})},
$$

depending on the fixed orientation $\mathbf{v}$. We have then a direction-dependent average chord length $\Lambda(\mathbf{v})=\int \ell \mathscr{P}(\ell \mid \mathbf{v}) d \ell=1 / \rho(\mathbf{v})$. For the special case of isotropic tessellations, $d H=d U$, and the integral in Eq. (30) yields

$$
\int_{\Omega_{d}^{+}}|\mathbf{n} \cdot \mathbf{v}| d U(\mathbf{n})=\frac{2}{\alpha_{d}},
$$


so that we recover $\rho(\mathbf{v})=\rho$.

\subsubsection{Line sections and the Cauchy formula}

We will now examine the case where the lines are random. Consider a bounded domain $X$ in $\mathbb{R}^{d}$ and an ensemble of homogeneous and isotropic random lines $\mathscr{T}$ (an explicit construction for $\mathscr{T}$ is given in Appendix A). The probability that a line drawn from this ensemble hits a sub-domain $Y \subset X$ is given by

$$
P(\mathscr{T} \cap Y \neq 0)=\frac{S_{d}(Y)}{S_{d}(X)},
$$

305 where $S_{d}(A)$ denotes the surface of a domain $A$ in $\mathbb{R}^{d}$ (Miles, 1972b). This result is independent of the position and orientation of the sub-domain $Y$ within $X$. Let us denote by $\ell$ the random line sections induced on $X$. The Cauchy theorem (also called Crofton second theorem) states that the ensemble average $\mathbb{E}[\ell]$ of the random line sections satisfies

$$
\mathbb{E}[\ell]=\alpha_{d} \frac{V_{d}(X)}{S_{d}(X)}
$$

310 where $V_{d}(A)$ denotes the volume of a domain $A$ in $\mathbb{R}^{d}$, provided that lines are homogeneous and isotropic (Miles, 1972b, 1986). In other words, the average line section $\mathbb{E}[\ell]$ through $X$ depends only on the ratio between the volume and the surface of $X$, regardless of the specific details of the shape of the domain, up to the dimension-dependent constant $\alpha_{d}$. This is a special case of a broader set of so-called stereological formulas derived by Miles for the projection of a body (or a collection of bodies) onto random $s$-dimensional flats in $\mathbb{R}^{d}$ (Miles, 1972b, 1985, 1986).

Consider then a disjoint aggregate of convex domains $\left\{X_{i}\right\} \subset X, i=1,2, \cdots, Q$, in $\mathbb{R}^{d}$. From Eqs. (33) and (34), the average line section through the aggregate 320 reads

$$
\mathbb{E}\left[\sum_{j=1}^{q} \ell_{j}\right]=\alpha_{d} \sum_{i=1}^{Q} \frac{V_{d}\left(X_{i}\right)}{S_{d}(X)}
$$

where $\ell_{j}, j=1, \cdots, q$ are the line sections of the non void intersections $\mathscr{T} \cap X_{j}$ between the lines and the domains composing the aggregate (Miles, 1972b). The number $q$ of such intersections is itself random, with expected value

$$
\mathbb{E}[q]=\sum_{\substack{i=1 \\ 12}}^{Q} \frac{S_{d}\left(X_{i}\right)}{S_{d}(X)} .
$$




\begin{tabular}{ccc}
\hline \hline Law $H(\mathbf{n})$ & $\zeta_{3}$ & $\eta_{3}$ \\
\hline Isotropic & $\pi / 8 \simeq 0.3927$ & 8 \\
Quadratic & $0.3029 \pm 2 \times 10^{-5}$ & $10.2368 \pm 5 \times 10^{-5}$ \\
Box & $2 / 9 \simeq 0.2222$ & $27 / \pi \simeq 8.59436$ \\
Histogram & $0.1880 \pm 3 \times 10^{-5}$ & $16.597 \pm 2 \times 10^{-5}$ \\
\hline \hline
\end{tabular}

Table 1: Numerical values for the constants $\zeta_{3}$ (see Eq. (14)) and $\eta_{3}$ (see Eq. (17)), for $d=3$ and various laws $H(\mathbf{n})$. Integrals have been computed by Monte Carlo sampling for quadratic and histogram laws (whence the error bars) and analytically for isotropic and box laws.

By formally taking the ratio between Eqs. (35) and (36), the average line section for the domains composing the aggregate $\left\{X_{i}\right\}$ can be estimated by

$$
\lambda \equiv \frac{\mathbb{E}\left[\sum_{j=1}^{q} \ell_{j}\right]}{\mathbb{E}[q]}=\alpha_{d} \frac{\sum_{i=1}^{Q} V_{d}\left(X_{i}\right)}{\sum_{i=1}^{Q} S_{d}\left(X_{i}\right)} .
$$

Suppose now that the aggregate described above is a Poisson stochastic tessellation in $\mathbb{R}^{d}$, whose cells are disjoint, space-filling polyhedra. In the limit of infinite size, averages over the constituents of the geometries can be replaced by ensemble averages over the random realizations of the tessellation (Miles, 1972b, 1986). In particular, for the average volume of a cell we have

$$
\lim _{Q \rightarrow \infty} \frac{1}{Q} \sum_{i=1}^{Q} V_{d}\left(X_{i}\right) \rightarrow\left\langle V_{d}\right\rangle=\frac{d !}{\zeta_{d}}\left(\frac{2}{\alpha_{d} \rho}\right)^{d}
$$

and for the average surface of a cell we have

$$
\lim _{Q \rightarrow \infty} \frac{1}{Q} \sum_{i=1}^{Q} S_{d}\left(X_{i}\right) \rightarrow\left\langle S_{d}\right\rangle=\alpha_{d} \rho\left\langle V_{d}\right\rangle
$$

Finally, combining Eqs. (37), (38) and (38), the average chord length $\Lambda$ through the cells of the tessellation induced by the random lines $\mathscr{T}$ can be estimated from

$$
\Lambda \equiv \lim _{Q \rightarrow \infty} \lambda \rightarrow \alpha_{d} \frac{\left\langle V_{d}\right\rangle}{\left\langle S_{d}\right\rangle}=\frac{1}{\rho}
$$

provided that the lines are uniformly and isotropically distributed (Miles, 1972b). This remarkable result show that under these hypotheses the average chord length $\Lambda$ for anisotropic Poisson tessellations of infinite size is simply equal to the inverse of the tessellation density $\rho$, and does not depend on the anisotropy law 


\begin{tabular}{ccc}
\hline \hline Law $H(\mathbf{n})$ & $\left\langle f_{3}\right\rangle$ & Monte Carlo \\
\hline Isotropic & 6 & $6 \pm 5 \times 10^{-7}$ \\
Quadratic & 6 & $6 \pm 7 \times 10^{-7}$ \\
Box & 6 & $6 \pm 0$ \\
Histogram & 6 & $6 \pm 9 \times 10^{-7}$ \\
\hline \hline
\end{tabular}

Table 2: Average number of faces $\left\langle f_{3}\right\rangle$ of the Poisson tessellation cells for a box of side $L=120$, with various anisotropy laws $H(\mathbf{n})$, for $d=3$. The tessellation density is $\rho=1$ for all the angular laws $H(\mathbf{n})$. The limit value for infinite Poisson tessellations is $\left\langle f_{3}\right\rangle=6$ for any $H(\mathbf{n})$.

\begin{tabular}{ccc}
\hline \hline Law $H(\mathbf{n})$ & $\left\langle V_{3}\right\rangle$ & Monte Carlo \\
\hline Isotropic & $6 / \pi \simeq 1.90986$ & $1.90 \pm 0.01$ \\
Quadratic & $\simeq 2.4744$ & $2.44 \pm 0.02$ \\
Box & $27 / 8=3.375$ & $3.37 \pm 0.02$ \\
Histogram & $\simeq 3.987$ & $3.93 \pm 0.03$ \\
\hline \hline
\end{tabular}

Table 3: Average volume $\left\langle V_{3}\right\rangle$ of the Poisson tessellation cells for a box of side $L=120$, with various anisotropy laws $H(\mathbf{n})$, for $d=3$. The tessellation density is $\rho=1$ for all the angular laws $H(\mathbf{n})$. The limit value for infinite Poisson tessellations is given in Eq. (13).

$H(\mathbf{n})$ (although $V_{d}\left(X_{i}\right)$ and $S_{d}\left(X_{i}\right)$ separately depend on $H(\mathbf{n})$ ). Observe that the definition in Eq. (40) formally corresponds to taking

$$
\frac{1}{\Lambda}=\int_{\Omega_{d}^{+}} \rho(\mathbf{v}) d U(\mathbf{v})=\rho
$$

i.e., averaging the Poisson density $\rho(\mathbf{v})$ as in Eq. (30) over the uniform angular distribution $U(\mathbf{v})$.

The quantity $\Lambda$ physically represents the typical correlation length of the tessellation, i.e., the spatial scale of the local heterogeneity (Pomraning, 1991a). Since the observables related to particle transport in stochastic tessellations are mostly affected by the chord length distribution (and in particular by its average $\Lambda$ ), Eq. (40) might lead to conclude that the effects of anisotropy will come into play for $\Lambda \Sigma_{t} \simeq 1$, i.e., when the neutron mean free path $1 / \Sigma_{t}\left(\Sigma_{t}\right.$ being the total cross section) is comparable to the size of the random chunks. For $\Lambda \Sigma_{t} \ll 1$, particle transport should become less sensitive to the finer details of the tessellation due to the angular law $H(\mathbf{n})$. 


\begin{tabular}{ccc}
\hline \hline Law $H(\mathbf{n})$ & $\left\langle V_{3}^{2}\right\rangle$ & Monte Carlo \\
\hline Isotropic & 48 & $47.3 \pm 0.6$ \\
Quadratic & $\simeq 79.6227$ & $77.9 \pm 1.0$ \\
Box & $729 / 8=91.125$ & $91.5 \pm 1.3$ \\
Histogram & $\simeq 207.9931$ & $202 \pm 3$ \\
\hline \hline
\end{tabular}

Table 4: Second moment of the volume $\left\langle V_{3}^{2}\right\rangle$ of the Poisson tessellation cells for a box of side $L=120$, with various anisotropy laws $H(\mathbf{n})$, for $d=3$. The tessellation density is $\rho=1$ for all the angular laws $H(\mathbf{n})$. The limit value for infinite Poisson tessellations is given in Eq. (16).

\section{Monte Carlo investigation of Poisson tessellations}

We have developed a computer code that is capable of generating an ensemble of anisotropic Poisson geometries based on the procedure described above. This set of geometries can be then exported and used by a Monte Carlo code in order to produce reference solutions for particle transport within random media. Due to the complexity of the involved construction algorithms, for the case of isotropic tessellations extensive verifications have been previously carried out in order to verify that the generated tessellations obeyed the expected statistical properties (Larmier et al., 2016). In view of the novelty of the anisotropy laws, before addressing particle transport we will similarly verify the statistical properties of anisotropic Poisson tessellations by Monte Carlo simulation. For this purpose, the observables of interest, including the volume and surface of the cells, the number of faces per cell, and the chord length, are recorded for each realization. Once the set has been generated, ensemble averages (and possibly also full distributions, if the number of realizations is large enough) are estimated. In the following, we illustrate some significant examples as a function of the angular law $H(\mathbf{n})$ in dimension $d=3$, which is relevant for physical applications.

\subsection{Choice of the angular distributions}

In dimension $d=3$, the direction (unit) vector $\mathbf{n}$ can be characterized by assigning two angles, namely the co-latitude $\theta$ and the azimuth $\phi$ (see Fig. 1). We have then $d H(\mathbf{n})=d H(\theta, \phi)$, or $d H(\mathbf{n})=d H(\mu, \phi)$ when using the cosine $\mu=\cos (\theta)$. The isotropic distribution in $\Omega_{3}^{+}$yields

$$
H_{\text {iso }}(\theta, \phi)=\frac{1}{2 \pi} \sin (\theta)
$$

with $0 \leq \theta<\pi$ and $0 \leq \phi<\pi$. In order to probe the effects of the angular distribution, we introduce a few examples of anisotropy laws that might mimic the 
effects of stratification along the $z$ axis. In other words, we will preferentially sample planes whose normal vector is parallel to the $z$ axis. For the sake of simplicity, we will assume that the distribution $H(\mathbf{n})$ can be factorized with respect to the two variables, and that the distribution of $\phi$ is uniform (in other words, we preserve the invariance by rotation around the $z$ axis). A quadratic anisotropy can be introduced in the form

$$
H_{\text {quadratic }}(\mu, \phi)=\frac{3}{2 \pi} \mu^{2} \text { for }-1 \leq \mu<1,
$$

which has its minimum in $\mu=0$ and the maxima in $\mu= \pm 1$. A general case that might be of interest for applications is a piece-wise constant distribution, e.g.,

$$
H_{\text {histogram }}(\mu, \phi)=\frac{1}{A} \times \begin{cases}80 & \text { for }-1 \leq \mu<-0.95 \\ 4 & \text { for }-0.95 \leq \mu<-0.5 \\ 2 & \text { for }-0.5 \leq \mu<-0.25 \\ 1 & \text { for }-0.25 \leq \mu<0,\end{cases}
$$

and symmetric in the range $0<\mu<1$, which has maxima around $\mu= \pm 1$. The normalization constant for this example reads $A=13.1$. More complex functional forms for $H(\mathbf{n})$ can be easily conceived. For the special case of PoissonBox tessellations with three fixed orientations parallel to the orthogonal Cartesian axes we have

$$
\begin{gathered}
H_{\mathrm{box}}(\theta, \phi)=\frac{1}{3} \delta(\phi) \delta\left(\theta-\frac{\pi}{2}\right) \\
+\frac{1}{3} \delta\left(\phi-\frac{\pi}{2}\right) \delta\left(\theta-\frac{\pi}{2}\right)+\frac{1}{3} \delta(\theta) \frac{1}{\pi} .
\end{gathered}
$$

For the functional forms of the angular distribution $H(\mathbf{n})$ introduced here, the integrals needed for the constants $\zeta_{3}$ (see Eq. (14)) and $\eta_{3}$ (see Eq. (17)) can be easily computed by Monte Carlo sampling, since they are both expressed as expected values over the angular distributions. The functionals to be evaluated are the volume of the parallelepiped spanned by three unit vectors in the case of $\zeta_{3}$, and the scalar product of two unit vectors in the case of $\eta_{3}$, respectively. In special cases, such as for the isotropic and box distributions, the integrals can be computed analytically. The resulting constants $\zeta_{3}$ and $\eta_{3}$ are provided in Tab. 1 . Finally, observe that from Eq. (1) we have $\alpha_{3}=4$.

\subsection{Polyhedral features}

In order to explore the statistical features of the cells of the tessellations, we have generated anisotropic Poisson tessellations of a three-dimensional box of 


\begin{tabular}{ccc}
\hline \hline Law $H(\mathbf{n})$ & $\left\langle S_{3}\right\rangle$ & Monte Carlo \\
\hline Isotropic & $24 / \pi \simeq 7.63944$ & $7.60 \pm 0.03$ \\
Quadratic & $\simeq 9.986$ & $9.79 \pm 0.04$ \\
Box & $27 / 2=13.5$ & $13.50 \pm 0.06$ \\
Histogram & $\simeq 15.947$ & $15.75 \pm 0.07$ \\
\hline \hline
\end{tabular}

Table 5: Average surface $\left\langle S_{3}\right\rangle$ of the Poisson tessellation cells for a box of side $L=120$, with various anisotropy laws $H(\mathbf{n})$, for $d=3$. The tessellation density is $\rho=1$ for all the angular laws $H(\mathbf{n})$. The limit value for infinite Poisson tessellations is given in Eq. (10).

side $L$. For each angular law $H(\mathbf{n})$ described above, based on our computer code we have obtained a large number of realizations for increasing sides $L$ and the same tessellation density $\rho=1$. The side of the box is varied between $L=1$ and $L=120$ : we expect that when $L \rho \gg 1$ finite-size effects fade away and the moments of the observables converge to their asymptotic limits corresponding to infinite tessellations. We have generated $5 \times 10^{3}$ realizations for $1 \leq L \leq 30$, $2 \times 10^{3}$ realizations for $40 \leq L \leq 80$ and $10^{3}$ realizations for $100 \leq L \leq 120$. The Monte Carlo simulation results for the average number of faces $\left\langle f_{3}\right\rangle$ of each cell are given in Tab. 2 for the largest tested box, i.e., $L=120$ : it is immediately apparent that $\left\langle f_{3}\right\rangle$ does not depend on the angular law $H(\mathbf{n})$, as expected.

The simulation results for the average volume $\left\langle V_{3}\right\rangle$, the second moment $\left\langle V_{3}^{2}\right\rangle$, the average surface $\left\langle S_{3}\right\rangle$ and the correlations $\left\langle V_{3} S_{3}\right\rangle$ between the volume and the surface are given in Tabs. 3-6, respectively, for $L=120$. Numerical findings are in good agreement with the theoretical formulas, and the minimum of all these quantities attained for the case of isotropic tessellations, as predicted by theory. The evolution of these moments as a function of the side $L$ of the box is shown in Figs. 3 and 4: for small $L$, finite-size effects are clearly visible; for larger $L$, the estimated moments converge to their asymptotic limits for infinite tessellations. The rate of convergence to the asymptotic values depends on the anisotropy law $H(\mathbf{n})$ : the stronger the anisotropy, the slower the convergence. Isotropic and box tessellations, although converging to different limits, appear to have the nearly same rate of convergence, which stems from box tessellations being quasi-isotropic. Inspection of Fig. 3 shows that $\left\langle V_{3}\right\rangle$ and $\left\langle V_{3}^{2}\right\rangle$ appear to have similar rates of convergence, for a given $H(\mathbf{n})$.

The distribution of the inradius $r_{\text {in }, 3}$ has been also estimated by Monte Carlo simulation and is provided in Fig. 5 for large $L=120$ and $\rho=1$. It is immediately apparent that the curves corresponding to different laws $H(\mathbf{n})$ collapse onto the same functional form, which for infinite tessellations is the exponential 


\begin{tabular}{ccc}
\hline \hline Law $H(\mathbf{n})$ & $\left\langle V_{3} S_{3}\right\rangle$ & Monte Carlo \\
\hline Isotropic & 96 & $94.8 \pm 0.9$ \\
Quadratic & $\simeq 159.2455$ & $156 \pm 2$ \\
Box & $729 / 4=182.25$ & $183 \pm 3$ \\
Histogram & $\simeq 415.9862$ & $405 \pm 5$ \\
\hline \hline
\end{tabular}

Table 6: Volume-surface correlations $\left\langle V_{3} S_{3}\right\rangle$ of the Poisson tessellation cells for a box of side $L=120$, with various anisotropy laws $H(\mathbf{n})$, for $d=3$. The tessellation density is $\rho=1$ for all the angular laws $H(\mathbf{n})$. The limit value for infinite Poisson tessellations is given in Eq. (11).

distribution

$$
\mathscr{P}\left(r_{\mathrm{in}, 3}\right)=4 \rho e^{-4 \rho r_{\mathrm{in}, 3}},
$$

\section{A model of assembly with fragmented fuel pins}

We will now apply the anisotropic Poisson tessellations described above to the analysis of criticality-safety problems related to fuel degradation. For this 


\begin{tabular}{ccc}
\hline \hline$H(\mathbf{n})$ & $\Lambda$ & Monte Carlo \\
\hline Isotropic & 1 & $0.992 \pm 3 \times 10^{-3}$ \\
Quadratic & 1 & $0.991 \pm 9 \times 10^{-3}$ \\
Box & 1 & $0.992 \pm 1 \times 10^{-3}$ \\
Histogram & 1 & $0.990 \pm 1 \times 10^{-2}$ \\
\hline \hline
\end{tabular}

Table 7: Average chord lengths $\Lambda$ of the Poisson tessellation cells for a box of side $L=120$, with various anisotropy laws $H(\mathbf{n})$, for $d=3$. The tessellation density is $\rho=1$ for all the angular laws $H(\mathbf{n})$. The line orientation is uniform and isotropic. The limit value for infinite Poisson tessellations is $\Lambda=1 / \rho$, as in Eq. (40).

purpose, we will revisit the simple benchmark model introduced in (Larmier et al., 2018a), which we briefly recall in the following in order for this paper to be self-contained. As a reference configuration we will consider an assembly composed of $17 \times 17$ square fuel pin-cells of side length $\delta=1.262082 \mathrm{~cm}$ in the plane $O_{x y}$ and of height $L_{z}=10 \mathrm{~cm}$. Reflective boundary conditions are imposed on all sides of the assembly. The fuel elements will be entirely either of the UOX or MOX type: the respective material compositions and temperatures, corresponding to Beginning Of Life (i.e., non-depleted) fuel, are the same as in (Larmier et al., 2018a).

In order to describe a melted fuel assembly with random material fragmentation, we assume that the fuel lattice is replaced by a Poisson tessellation. The effects of material stratification will be modelled by introducing an anisotropy law $H(\theta, \phi)$ giving rise to planes that are preferentially orthogonal to the $z$ axis. The typical size of the fuel fragments will be imposed by setting the average chord length $\Lambda$, which is a free parameter of the model (related to the tessellation density by $\rho=1 / \Lambda$ ). For our benchmark, we have assumed that the assembly is composed of only three materials (fuel, cladding and moderator), which leads to a ternary stochastic mixture: each polyhedron of the Poisson tessellation is assigned a material composition by attributing a 'color', namely, ' $\mathscr{F}$ ' for fuel, ' $\mathscr{C}$ ' for cladding and ' $\mathscr{M}$ ' for moderator. The corresponding coloring probabilities $p_{\mathscr{F}}, p_{\mathscr{C}}$ and $p_{\mathscr{M}}=1-p_{\mathscr{F}}-p_{\mathscr{C}}$ are chosen so that for each material $i$ the ensemble-averaged volumic ratio $\left\langle p_{i}\right\rangle$ coincides with that of a pin-cell before fragmentation:

$$
\begin{gathered}
p_{\mathscr{F}}=\pi R_{1}^{2} / \delta^{2} \approx 0.335861 \\
p_{\mathscr{C}}=\pi\left(R_{2}^{2}-R_{1}^{2}\right) / \delta^{2} \approx 0.107943 \\
p_{\mathscr{M}}=\left(\delta^{2}-\pi R_{2}^{2}\right) / \delta^{2} \approx 0.556196
\end{gathered}
$$

where $R_{1}$ is the outer radius of the fuel pellet and $R_{2}$ is the outer radius of the 19 
cladding. For illustration, an example of assembly configurations before and after fuel degradation is shown in Fig. 7. For each realization, the volumic ratio of material $i$ in the tessellation is not precisely equal to $p_{i}$, because of finite-size effects. Imposing mass preservation in n-nary statistical mixing models would be non-trivial: in this respect, the lack of strict mass conservation is a limitation of standard colored stochastic tessellations. However, the impact of finite-size effects decreases with decreasing $\Lambda$ : for a thorough discussion, see, e.g., (Larmier et al., 2018a). It is important to stress that the resulting material regions (i.e., the clusters composed of all adjacent polyhedral cells sharing the same color) are generally non-convex. However, the the average chord length $\Lambda_{i}(\mathbf{v})$ through clusters with label $i$ is simply related to the average chord length $\Lambda(\mathbf{v})$ of the un-colored cells via $\Lambda(\mathbf{v})=\left(1-p_{i}\right) \Lambda_{i}(\mathbf{v})$, which follows from the binomial distribution of the coloring procedure (Larmier et al., 2016).

Similarly as done in (Larmier et al., 2018a), for our benchmark configuration we will investigate the behaviour of the ensemble-averaged multiplication factor $\left\langle k_{e f f}\right\rangle$ and the ensemble-averaged kinetics parameters, namely, the effective neutron generation time $\left\langle\Lambda_{\text {eff }}\right\rangle^{2}$ and the effective delayed neutron fraction $\left\langle\beta_{\text {eff }}\right\rangle$. The ensemble-averaged scalar particle flux $\langle\varphi(E)\rangle$ within the assembly will be also examined.

In particular, we will quantify the effects of the fuel fragmentation on these observables in terms of both the anisotropy law $H(\mathbf{n})$ and the average chord length $\Lambda$. For each $\Lambda$, we will test the impact of material stratification by varying the law $H(\mathbf{n})$, from isotropic to strongly anisotropic. In particular, we have considered the quadratic, histogram and box anisotropy laws introduced in Sec. 3.1 (the respective parameters are the same as those given above). The limit case of slab tessellations, where the random planes (with exponentially distributed distances) are exactly orthogonal to the $z$ axis, will be also explored for comparison. Some example of realizations corresponding to different anisotropy laws are shown in Fig. 8. As for the typical fragment size, in-core experiments suggest that $\Lambda$ might lie in the range from less than a millimetre to several millimetres, depending on the re-solidification speed (Hagen and Hofmann, 1987; Broughton et al., 1989; Hofmann, 1999). For each law $H(\mathbf{n})$ we will then analyse the effects of $\Lambda$ on the observables. For $\Lambda$ comparable to the linear size of the assembly, the tessellations are dominated by finite-size effects, and the dispersion of the volumic ratio for each material composition becomes large. For small $\Lambda$ (the so-called atomic mix limit), the effects of the anisotropy law $H(\mathbf{n})$ are expected

\footnotetext{
${ }^{2}$ The symbol $\left\langle\Lambda_{e f f}\right\rangle$ for the average neutron generation time is not to be confused with $\Lambda$, which denotes the average chord length.
} 
to become less discernible with respect to the isotropic case; moreover, the com-

\subsection{Average multiplication factor}

The behaviour of the ensemble-averaged multiplication factor $\left\langle k_{\text {eff }}\right\rangle$ is illustrated in Fig. 9 for UOX (left) and MOX (right) assemblies. We have determined the evolution of $\left\langle k_{e f f}\right\rangle$ as a function of the anisotropy laws (quadratic, 
box, histogram and slab) and of the average chord length $\Lambda$. Simulation results corresponding to isotropic Poisson tessellations had been previously examined in (Larmier et al., 2018a) and are reported here for comparison. The error bar on $\left\langle k_{\text {eff }}\right\rangle$ stems from the contribution of the Monte Carlo statistical error (which is of the order of about $50 \mathrm{pcm}$ for all simulations, as mentioned above) and the dispersion due to the random realizations. For reference, the limit case of atomic $\operatorname{mix}(\Lambda \rightarrow 0)$ and the $k_{\text {eff, },}$ eigenvalue corresponding to an assembly with intact fuel pins are also shown in Fig. 9.

Fuel fragmentation has an apparent impact on stochastic tessellations. For both UOX and MOX assemblies, in the atomic mix limit $\left\langle k_{\text {eff }}\right\rangle$ lies well below the reference $k_{\text {eff, },}$ (about $8 \times 10^{3} \mathrm{pcm}$ for UOX and $1.2 \times 10^{4} \mathrm{pcm}$ for MOX). Close to the atomic mix limit, i.e., $\Lambda \rightarrow 0$, we can single out two general trends: the behaviour of $\left\langle k_{e f f}\right\rangle$ as a function of $\Lambda$ is almost independent of the anisotropy

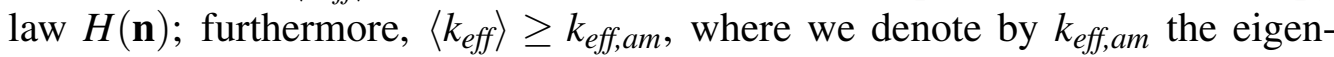
value corresponding to the atomic mix limit. This latter feature had been previously shown (via a perturbative approach) in a rod geometry with Poisson mixing statistics for mono-energetic transport (Pomraning, 1999, 1991b), based on earlier work (Williams, 1970, 1974). Their conclusions apparently hold true also for the three-dimensional, highly heterogeneous configurations considered here (independently of $H(\mathbf{n})$ ), although a word of caution is in order: the application of expectation operators to the eigenvalue equation might subtly affect the value of $\left\langle k_{\text {eff }}\right\rangle$ with respect to the deterministic $k_{\text {eff,am }}$ (Pomraning, 1999, 1991b; Williams, 1970, 1974). The former feature was expected on physical grounds, based on the considerations presented in Sec. 2.3.3: for $\Lambda$ smaller than the neutron mean free path, the distinct details of the angular law $H(\mathbf{n})$ are not detected by the transported particles, and $\left\langle k_{\text {eff }}\right\rangle$ is almost exclusively affected by the average chord length $\Lambda$, which is independent of $H(\mathbf{n})$. This result stresses the importance of $\Lambda$ as a key property of the Poisson tessellations with respect to particle transport.

For increasing $\Lambda$, the effects of the anisotropy $H(\mathbf{n})$ on $\left\langle k_{\text {eff }}\right\rangle$ become apparent: the curve of $\left\langle k_{\text {eff }}\right\rangle$ as a function of $\Lambda$ gets progressively lower when $H(\mathbf{n})$ is varied from isotropic to strongly anisotropic (the histogram distribution leading to an almost horizontal material stratification in the assembly). The curve of $\left\langle k_{\text {eff }}\right\rangle$ corresponding to the quadratic anisotropy, which is somehow milder than the histogram distribution, lies in between. The highest $\left\langle k_{e f f}\right\rangle$ value is attained for an isotropic $H(\mathbf{n})$, which is possibly related to the peculiar inequalities for the polyhedral features of the Poisson tessellation described in Sec. 2.2.3. The values $\left\langle k_{\text {eff }}\right\rangle$ computed for isotropic and box tessellations are almost indistin- 
guishable, as already noticed in our previous work (Larmier et al., 2018a): this surprising and important finding stems from the box tessellation being quasiisotropic. Numerical investigations have actually shown that the chord length distribution for Poisson-Box tessellations is almost identical to that of isotropic Poisson tessellations (Larmier et al., 2017b, 2018a).

In the limit of very large $\Lambda$, the stochastic tessellations are trivially filled with a single material (fuel, cladding, or moderator). In this case, $\left\langle k_{\text {eff }}\right\rangle$ would be the weighted sum of the multiplication factors of three configurations with the assembly replaced by a fuel, cladding or moderator volume, and this value would be typically lower than the reference $k_{\text {eff, },}$ corresponding to the regular lattice (Larmier et al., 2018a).

For any anisotropy law $H(\mathbf{n})$, the eigenvalue $\left\langle k_{\text {eff }}\right\rangle$ as a function of the average chord length $\Lambda$ attains a maximum, which can be in excess or in defect with respect to $k_{\text {eff, },}$, depending on the mixing statistics and on the material compositions, but only weakly on the angular law $H(\mathbf{n})$. For the UOX assemblies, the maximum $\left\langle k_{\text {eff }}\right\rangle$ is attained for $\Lambda \sim 0.5 \mathrm{~cm}$ (almost independently of $H(\mathbf{n})$ ), and lies slightly lower than $k_{\text {eff, },}$. For MOX assemblies, the maximum is attained at a larger average chord length $\Lambda \sim 1 \mathrm{~cm}$, again almost independently of $H(\mathbf{n})$, and is fairly in excess of the reference $k_{\text {eff, },}$. Fuel fragmentation would be expected to lower the reactivity of the assemblies, where fuel is arranged in a regular lattice with optimized pitch. This seems to be the case for UOX compositions, where the tessellation model leads to maxima $\left\langle k_{\text {eff }}\right\rangle<k_{\text {eff, } 0}$, independently of the angular law $H(\mathbf{n})$. In practice, neutron thermalization in the moderator will also induce spectral effects, and it turns out that MOX composition generally lead to larger reactivity excursions, with maxima well beyond $k_{\text {eff,o }}$ for any $H(\mathbf{n})$. As observed above, fissile mass is not rigorously preserved along realizations, and this might further contribute to the discrepancies between UOX and MOX assemblies for intermediate correlation lengths (see also (Larmier et al., 2018a)).

For both UOX and MOX configurations, the behaviour of $\left\langle k_{e f f}\right\rangle$ for slab tessellations is distinct with respect to the case of regular anisotropic Poisson tessellations. Slab tessellations formally correspond to degenerate anisotropic Poisson tessellations where a single direction for sampling the random planes is allowed: in the limit of infinite tessellations, this yields polyhedral cells of unbounded volume and surface (contrary to the case of non-degenerate $H(\mathbf{n})$ distributions, for which the polyhedral cells are always bounded, even for infinite tessellations). Nonetheless, the average chord length $\Lambda$ is well defined and stays finite, which allows comparing slab tessellations to anisotropic Poisson tessellations for a given $\Lambda$. Moreover, for finite tessellations as those applied to the fragmented 
assembly model, all polyhedral features of slab tessellations are finite, although cells span the entire geometry. Yet, the peculiar geometrical features of these configurations is mirrored in the resulting properties of the eigenvalues for criticality calculations: in particular, the maximum $\left\langle k_{\text {eff }}\right\rangle$ eigenvalue is attained for smaller $\Lambda$ (about $0.25 \mathrm{~cm}$ for UOX compositions, and $0.5 \mathrm{~cm}$ for MOX compositions) and the peak value is lower than in the case of regular anisotropic Poisson tessellations. For MOX assemblies, the maximum corresponding to slab tessellations lies in slight defect of $k_{\text {eff, },}$. These findings are intimately related to the fact that moderator and cladding layers in slab configurations allow for longer neutron excursions in the transversal direction (i.e., without encountering fuel layers) that are forbidden when the polyhedral cells are bounded.

\subsection{Distribution of the multiplication factor}

For criticality safety applications, the dispersion of the multiplication factors around their average values due to the variability of the random geometry realizations is of utmost interest. For this purpose, we have also computed the distributions $\mathscr{P}\left(k_{e f f}\right)$. Some representative histograms corresponding to MOX fuel are displayed in Fig. 10 as a function of the anisotropy law $H(\mathbf{n})$ for fixed $\Lambda$ (left) or as a function of $\Lambda$ for the same law $H(\mathbf{n})$ (right). In particular, Fig. 10 (left) shows that the shape of the $\mathscr{P}\left(k_{\text {eff }}\right)$ distribution is only weakly sensitive to the anisotropy law $H(\mathbf{n})$ for the value of $\Lambda=1 \mathrm{~cm}$ chosen here. Only the slab tessellation shows a largely different behaviour, as already suggested by the analysis of the average $\left\langle k_{e f f}\right\rangle$. All the distributions are highly skewed, with a longer tail for lower values of $k_{\text {eff. }}$. Figure 10 (right) shows the impact of the average chord length for the case of the histogram anisotropy distribution. It is apparent that $\Lambda$ strongly affects not only the average $\left\langle k_{\text {eff }}\right\rangle$, but also the dispersion and the skewness of the full distribution $\mathscr{P}\left(k_{e f f}\right)$.

\subsection{Average kinetics parameters}

TRIPOLI-4 ${ }^{\circledR}$ computes adjoint-weighted kinetics parameters based on the Iterated Fission Probability (IFP) method (see (Larmier et al., 2018a)). The evo-

655 lution of the ensemble-averaged effective delayed neutron fraction $\left\langle\beta_{\text {eff }}\right\rangle$ is illustrated in Fig. 11 for UOX (left) and MOX (right) assemblies. The values $\left\langle\beta_{\text {eff }}\right\rangle$ obtained by Monte Carlo simulation are displayed as a function of the average chord length $\Lambda$, for different anisotropy laws $H(\mathbf{n})$. The isotropic case is taken from (Larmier et al., 2018a). The findings corresponding to slab tessellations are shown as well. The limit case corresponding to the atomic mix is also displayed. Moreover, the $\beta_{\text {eff, } O}$ value corresponding to an assembly with intact fuel pins is plotted for reference. The impact of stochastic tessellations on $\left\langle\beta_{\text {eff }}\right\rangle$ is weak, 
and typically well within error bars. The behaviour of $\left\langle\beta_{\text {eff }}\right\rangle$ is almost unaffected by the choice of the anisotropy law $H(\mathbf{n})$. The case of slab tessellations yields results that are entirely compatible with those of regular anisotropic tessellations. For UOX assemblies we remark nonetheless that the random fragmentation introduces a slight bias on the average value, i.e., $\left\langle\beta_{\text {eff }}\right\rangle \leq \beta_{\text {eff, },}$, where $\beta_{\text {eff, },}$ is the reference value corresponding to an assembly with intact fuel pins. On the

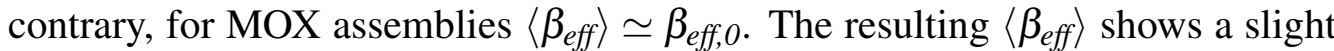
increasing trend with respect to the average chord length $\Lambda$. Actually, the effective delayed neutron fraction $\left\langle\beta_{\text {eff }}\right\rangle$ depends mostly on the volumic fraction of fuel within the assembly, and this quantity only weakly depends on $\Lambda$ and $H(\mathbf{n})$; however, for large $\Lambda$ finite-size effects come into play and affect the distribution of the fissile mass within the assembly (the mass is not exactly preserved by the ternary mixing model, as observed above).

The behaviour of the ensemble-averaged effective neutron generation time $\left\langle\Lambda_{\text {eff }}\right\rangle$ is illustrated in Fig. 12 for UOX (left) and MOX (right) assemblies. The values $\left\langle\Lambda_{\text {eff }}\right\rangle$ obtained by Monte Carlo simulation are displayed as a function of the average chord length $\Lambda$, for different anisotropy laws $H(\mathbf{n})$. The isotropic case is taken from (Larmier et al., 2018a). The findings corresponding to slab tessellations are shown as well. The limit case corresponding to the atomic mix is also displayed. Moreover, the $\Lambda_{e f f, O}$ value corresponding to an assembly with intact fuel pins is plotted for reference. Contrary to the delayed neutron fraction, the effective neutron generation time $\left\langle\Lambda_{e f f}\right\rangle$ is strongly affected by the fuel fragmentation. In the atomic mix limit for small $\Lambda,\left\langle\Lambda_{\text {eff }}\right\rangle$ lies slightly below $\Lambda_{\text {eff, } 0}$, and rapidly increases as a function of $\Lambda$. In particular, for UOX assemblies $\left\langle\Lambda_{\text {eff }}\right\rangle$ increases by a factor of 3 in the range of $\Lambda$ explored here, whereas for MOX assemblies $\left\langle\Lambda_{\text {eff }}\right\rangle$ increases by a factor of 6 for the same range of $\Lambda$. Enhancing the degree of anisotropy from the isotropic distribution to the histogram distribution increases $\left\langle\Lambda_{e f f}\right\rangle$, the quadratic law lying in between. Isotropic and box tessellations yield almost identical results. The simulation results corresponding to the slab tessellations are larger than for the regular anisotropic Poisson tessellations: these findings can be explained by the increased lifetime of neutrons in these configurations, the moderator and cladding layers allowing for longer excursions without being absorbed in the transversal directions, i.e, within the material layers.

\subsection{Analysis of neutron flux}

We consider now the effects of fuel fragmentation on the ensemble-averaged and normalized fundamental eigenmode $\langle\varphi(E)\rangle$. We have considered a 281 group mesh, covering the entire energy range of the simulation, namely $10^{-5} \mathrm{eV}$ 
to $20 \mathrm{MeV}$. The behaviour of the spatially-integrated and normalized $\langle\varphi(E)\rangle$ for MOX assemblies is shown in Fig. 13. For fixed $\Lambda$, the impact of the anisotropy law is rather weak, as shown in Fig. 13 (left), although spectra in tessellations clearly differ from those of the intact assembly and of the atomic mix. On the other hand, for a given anisotropy law $H(\mathbf{n})$, the impact of $\Lambda$ is apparent: for small $\Lambda,\langle\varphi(E)\rangle$ lies slightly below the reference flux (corresponding to the intact assembly) in the thermal region and above for the epi-thermal and fast regions. For larger $\Lambda,\langle\varphi(E)\rangle$ lies above the reference flux in the thermal region and below for the epi-thermal and fast regions.

\subsection{Simulation time}

Transport calculations have been run on a cluster based at CEA, with Intel Xeon E5-2680 V2 $2.8 \mathrm{GHz}$ processors. For all the configurations examined here, we have recorded the computational cost of the performed calculations as a function of the complexity of the underlying stochastic tessellations. The corresponding (ensemble-averaged) computer times for each assembly configuration are reported in Tab. 8, together with the associated dispersions $\sigma[t]$. The average simulation time $\langle t\rangle$ globally decreases for increasing $\Lambda$, as expected. The chosen anisotropy law $H(\mathbf{n})$ also affects the computer time. For large $\Lambda$, simulation times are almost independent of the anisotropy law; for small $\Lambda$, the shortest simulation times are attained for the slab tessellations. Moreover, subtle effects due to correlation lengths and volume fractions for the material compositions come also into play, and strongly influence the average computer time. For some configurations, the dispersion $\sigma[t]$ may become very large, and even be comparable to the average $\langle t\rangle$. Atomic mix simulations are based on a single homogenized realization, and the dispersion is thus trivially zero.

\section{Conclusions}

In this paper we have examined the application of anisotropic Poisson tessellations as an effective model for the analysis of criticality-safety problems. Building on a recent work, where we have shown that a broad class of stochastic tessellations can be used in order to describe the random fuel fragmentation and solidification following severe accidents (Larmier et al., 2018a), we have here focused on Poisson tessellations and we have addressed the effects of material stratification by including arbitrary anisotropy laws in the construction of the underlying geometries. This generalizes our previous findings, that were limited to isotropic tessellations. 


\begin{tabular}{cccc}
\hline \hline \multirow{2}{*}{ Fuel } & Anisotropy & $\begin{array}{c}\Lambda=0.15 \mathrm{~cm} \\
\langle\mathrm{t}\rangle \pm \sigma[\mathrm{t}]\end{array}$ & $\begin{array}{c}\Lambda=3 \mathrm{~cm} \\
\langle\mathrm{t}\rangle \pm \sigma[\mathrm{t}]\end{array}$ \\
\hline \multirow{6}{*}{ UOX } & Isotropic & $48260 \pm 16384$ & $2209 \pm 192$ \\
& Quadratic & $33691 \pm 7980$ & $2165 \pm 210$ \\
& Histogram & $19407 \pm 5560$ & $2185 \pm 234$ \\
& Box & $16451 \pm 346$ & $2165 \pm 198$ \\
& Slab & $5864 \pm 1737$ & $2643 \pm 821$ \\
\hline \multirow{4}{*}{ MOX } & Isotropic & $37628 \pm 8146$ & $2052 \pm 193$ \\
& Quadratic & $46726 \pm 28523$ & $2687 \pm 863$ \\
& Histogram & $19407 \pm 5560$ & $2185 \pm 234$ \\
& Box & $15033 \pm 3166$ & $1998 \pm 198$ \\
& Slab & $4031 \pm 1318$ & $1962 \pm 371$ \\
\hline \hline
\end{tabular}

Table 8: Average simulation times $\langle t\rangle$ (expressed in seconds) and their dispersions $\sigma[t]$ for UOX and MOX fuels, corresponding to different anisotropy laws $H(\mathbf{n})$, and average chord lengths $\Lambda$. For comparison, the simulation time for the atomic mix limit is $\mathrm{t}_{\text {mix }} \simeq 1350 \mathrm{~s}$ for UOX and $\mathrm{t}_{\text {mix }} \simeq 1200 \mathrm{~s}$ for MOX.

In order to better apprehend the behaviour of the anisotropic Poisson tessellations with respect to the physical observables that are of interest for particle transport, we have first analysed their key statistical features: the volume of a cell, its surface and number of faces, the inradius and the chord length distribution. Based on a random geometry generator developed at CEA, we have created large ensembles of realizations of three-dimensional Poisson tessellations corresponding to various anisotropy laws. For each law, we have examined the convergence of the Monte Carlo simulation results for the tessellation of finite-size boxes to the asymptotic limits that are known for infinite-size anisotropic tessellations. A remarkable feature is that the average chord length, which physically represents the typical correlation length of the geometry, does not depend on the anisotropy law. On the contrary, the average volume and surface of the cells of the tessellation depend on the anisotropy law, and their minimum is reached for the special case of isotropic tessellations.

Then, we have revisited a benchmark concerning eigenvalue calculations in randomly fragmented fuel assemblies (Larmier et al., 2018a). Starting from reference configurations with intact fuel pin cells, we have created ensembles of UOX and MOX configurations by using anisotropic Poisson tessellations with varying anisotropy laws and mean chord lengths. The anisotropy law was chosen in order to mimic the effects of stratification on the fuel degradation. The generated configurations were fed to the Monte Carlo transport code TRIPOLI- $4{ }^{\circledR}$, which has been used to compute the ensemble-averaged multiplication factor $\left\langle k_{e f f}\right\rangle$, the adjoint-weighted kinetics parameters $\left\langle\beta_{\text {eff }}\right\rangle$ and $\left\langle\Lambda_{e f f}\right\rangle$, and the scalar 
neutron flux $\langle\varphi(E)\rangle$. Anisotropy plays an important role in determining the behaviour of the physical observables related to fragmented configurations. However, when $\Lambda \rightarrow 0$, the neutron mean free path becomes large than the typical scale of the fragmentation, and the distinct effects of anisotropy fade away. MOX assemblies seem more sensitive than UOX assemblies to the perturbations introduced by the stochastic tessellations. The delayed neutron fraction $\left\langle\beta_{\text {eff }}\right\rangle$ is only weakly affected by random tessellations, whereas the multiplication factor $\left\langle k_{\text {eff }}\right\rangle$ and the neutron generation time $\left\langle\Lambda_{e f f}\right\rangle$ depend on both the anisotropy law and mean chord length. The maximum excursion of $\left\langle k_{\text {eff }}\right\rangle$ and the minimum excursion of $\left\langle\Lambda_{e f f}\right\rangle$ are reached in the case of isotropic tessellations, a result that is possibly related to the inequalities on the statistical features mentioned above.

The special case of box tessellations, that are quasi-isotropic, leads to transport properties that are almost identical to the isotropic case. Finally, the degenerate case of slab tessellations (leading to material layers that are exactly orthogonal to the $z$ axis) was separately considered, for comparison: it was shown that the transport properties are rather different than in the case of regular anisotropic Poisson tessellations, which can be understood based on the peculiar nature of the slab geometries, where neutrons can travel long distances along the transversal directions without being absorbed.

The results discussed in this paper provide a generalization of our previous findings for three-dimensional isotropic tessellations. Our approach is highly idealized and focused on methodological and conceptual aspects. Stochastic tessellations, despite being convenient model for disordered materials, does not fully qualify for providing an accurate description of reactor safety applications without further proof: in particular, multi-physics feedbacks such as temperature or density variations have been admittedly neglected in our analysis, and one might think of a variety of degraded configurations that cannot be represented by tessellations. Thus, the conclusions drawn in this paper may not directly apply to real-world problems, such as the assessment of re-criticality probability of outof-pile deposition of corium debris. Besides, other aspects of eigenvalue problems for neutron transport and multiplication in stochastic tessellations would deserve attention: for instance, it would be instructive to compare the reference solutions obtained in this work to the approximate solutions coming from the CLS model, in the spirit of what has been recently proposed for fixed-source transport problems (Larmier et al., 2018b,c). We conclude by observing that throughout this paper we have preserved the spatial homogeneity of the tessellations (while relaxing isotropy). Recent works have shown that it is possible to further extend the theoretical results for infinite Poisson tessellations to the 
case of spatially non-homogeneous configurations (Schneider and Weil, 2008; Schneider, 2013). The analysis of such class of tessellations, whose construction can be quite straightforwardly included in the Monte Carlo method described above, will be left for future research work.

\section{Acknowledgements}

TRIPOLI- ${ }^{\circledR}$ is a registered trademark of CEA. The authors wish to thank Électricité de France (EDF) for partial financial support. C. Larmier and A. Zoia express their gratitude to F. Malvagi and A. Mazzolo (CEA) and to E. Dumonteil (IRSN) for fruitful discussions.

\section{Appendix A. Plane and line sections}

In dimension $d=2$, a line section of a bounded domain $X \subset \mathbb{R}^{2}$ satisfying the homogeneity and isotropy property can be obtained as follows. Consider the disk $S$ containing $X$ and with the smallest possible radius. Take then an isotropic direction $\mathbf{v}$ emanating from the center of $S$. Take a random point whose position $z$ is uniformly distributed on the diameter of $S$ in direction $\mathbf{v}$. Construct now the line passing through $z$ and orthogonal to $\mathbf{v}$. If the line intersects the domain $X$, this is an acceptable line section satisfying the properties above; if not, repeat the whole procedure until a new line hits $X$.

815 In dimension $d=3$, a line section of a bounded domain $X \subset \mathbb{R}^{3}$ satisfying the homogeneity and isotropy property can be obtained by first constructing a plane section. Consider the sphere $S$ containing $X$ and with the smallest possible radius. Take then an isotropic direction $\mathbf{v}$ emanating from the center of $S$. Take a random point whose position $z$ is uniformly distributed on the diameter of $S$ in direction $\mathbf{v}$. Construct now the plane passing through $z$ and orthogonal to $\mathbf{v}$. If the plane intersects the domain $X$, this is a plane section of $X$ satisfying homogeneity and isotropy; if not, repeat the whole procedure until a new plane hits $X$. Once the plane section is obtained, the procedure for line sections in $d=2$ can be applied: it can be shown that an homogeneous and isotropic line section of an homogeneous and isotropic plane section of $X$ is actually an homogeneous and isotropic line section of $X$ in $d=3$.

Other procedures exist for homogeneous and isotropic line sections in dimension $d$ that do not require rejection methods (Coleman, 1969). This can be achieved, e.g., by first choosing a point $\mathbf{P}$ uniformly on the surface of $X \subset \mathbb{R}^{d}$ and then sampling lines passing through $\mathbf{P}$ and with direction $\mathbf{v}$ satisfying an isotropic incident flux with respect to the normal $\mathbf{n}$ entering the surface of $X$ at 
point $\mathbf{P}$. This condition imposes the so-called $\mu$-randomness measure (Santalo, 1976; Coleman, 1969)

$$
\alpha_{d} \frac{d \mathbf{v}}{\Omega_{d}} \frac{d S_{d}(X)}{S_{d}(X)}(\mathbf{v} \cdot \mathbf{n})
$$

The term $\cos \theta=\mathbf{v} \cdot \mathbf{n}$ implies that in polar coordinates trajectories starting on the surface must enter the domain $X$ with $\theta=\arcsin (2 \xi-1)$ in two dimensions and $\theta=1 / 2 \arccos (1-2 \xi)$ in three dimensions, $\xi$ being uniformly distributed in $(0,1]$ (Zoia et al., 2012a).

\section{References}

Adams, M.L., Larsen, E.W., Pomraning, G.C., 1989. J. Quant. Spectrosc. Radiat. Transfer 42, 253-266

Ambos, A.Yu., Mikhailov, G.A., 2011. Russ. J. Numer. Anal. Math. Modelling 26, 263-273.

Brantley, P.S., Palmer, T.S., 2009. In Proceedings of the international conference on mathematics, computational methods \& reactor physics (M\&C2009), Saratoga Springs, New York.

Brantley, P.S., 2009. In Proceedings of the international conference on mathematics, computational methods \& reactor physics (M\&C2009), Saratoga Springs, New York.

Brantley, P.S., 2011. J. Quant. Spectrosc. Radiat. Transfer 112, 599-618.

Broughton, J.M., Kuan, P., Petti, D.A., Tolman, E.L., 1989. Nucl. Techn. 87, 34-53.

Brown, F.B., Martin, W.R., 2004. Ann. Nucl. Energy 31, 2039-2047.

Brun, E., et al., 2015. Ann. Nucl. Energy 82, 151-160.

Chiu, S.N., Stoyan, D., Kendall, W.S., Mecke, J., 2013. Stochastic geometry and its applications, Wiley, New York, USA.

Coleman, R., 1969. J. Appl. Probab. 6, 430-441.

Donovan, T.J., Danon, Y., 2003. Nucl. Sci. Eng. 143, 226-239.

Donovan, T.J., Sutton, T.M., Danon, Y., 2003. In Proceedings of the nuclear mathematical and computational sciences: a century in review, a century anew, Gatlinburg, TN.

Doub, W.B., 1961. Nucl. Sci. Eng. 10, 299-307.

Gilbert, E.N., 1962. Ann. Math. Statist. 33, 958-972.

Goudsmit, S., 1945. Rev. Mod. Phys. 17, 321.

Griesheimer, D.P., Millman, D.L., Willis, C.R., 2010. J. Quant. Spectrosc. Radiat. Transfer 112, 577598.

Gunji, S., Tonoike, K., Izawa, K., Sono, H., 2017. Prog. Nuc. Energy 101, 321-328.

Hagen, S., Hofmann, P., 1987. Nucl. Eng. Design 103, 85-106.

Hofmann, P., 1999. J. Nucl. Mat. 270, 194-211.

Ji, W., Martin, W.R., 2011. Nucl. Sci. Eng. 169, 19-39.

865 Larmier, C., Dumonteil, E., Malvagi, F., Mazzolo, A., Zoia, A., 2016. Phys. Rev. E 94, 012130.

Larmier, C., Hugot, F.-X., Malvagi, F., Mazzolo, A., Zoia, A., 2017a. J. Quant. Spectrosc. Radiat. Transfer 189, 133148.

Larmier, C., Zoia, A., Malvagi, F., Dumonteil, E., Mazzolo, A., 2017b. J. Quant. Spectrosc. Radiat. Transfer. 196, 270-286.

870 Larmier, C., Zoia, A., Malvagi, F., Dumonteil, E., Mazzolo, A., 2018a. Ann. Nucl. Energy 111, 391-406. 
Larmier, C., Lam A., Brantley, P., Malvagi, F., Palmer, T., Zoia, A., 2018b. J. Quant. Spectrosc. Radiat. Transfer. 204, 256271.

Larmier, C., Zoia, A., Malvagi, F., Dumonteil, E., Mazzolo, A., 2018c. J. Quant. Spectrosc. Radiat. Transfer. 206, 70-82.

Larsen, E.W., Vasques, R., 2011. J. Quant. Spectrosc. Radiat. Transfer. 112, 619-631.

Lepage, T., Delaby, L., Malvagi, F., Mazzolo, A., 2011. Prog. Nucl. Sci. Techn. 2, 743-748.

Levermore, C.D., Pomraning, G.C., Sanzo, D.L., Wong, J., 1986. J. Math. Phys. 27, 2526-2536.

Liang, C., Ji, W., Brown, F.B., 2013. Ann. Nucl. Energy 53, 140-146.

880 Liang, C., Ji, 2011. Trans. Am. Nucl. Soc. 105, 495-497.

Malvagi, F., Byrne, R.N., Pomraning, G.C., Somerville, R.C.J., 1992. J. Atm. Sci. 50, 2146-2158.

McLane, V., 2004. Brookhaven National Laboratory report BNL-NCS-44945-01/04-REV KB0301042.

Mecke, J., 1995. Adv. Appl. Prob. 27, 56-62.

885 Meijering, J.L., 1953. Philips Res. Rep. 8, 270-290.

Miles, R. E., 1964. Proc. Nat. Acad. Sci. USA 52, 901-907.

Miles, R.E., 1970. Izv. Akad. Nauk. Arm. SSR Ser. Mat. 5, 263-285.

Miles, R.E., 1972. Suppl. Adv. Appl. Prob. 4, 243-266.

Miles, R.E., 1972. J. Microscopy 95, 181-195.

890 Miles, R.E., 1985. J. Microscopy 138, 115-125.

Miles, R.E., 1986. In: Science on form. Proceedings of the first international symposium for science on form, S. Ishizaka general editor, 147-155, KTK Scientific Publisher, Tokyo.

Murata, I., Mori, T., Nakagawa, M., 1996. Nucl. Sci. Eng. 123, 96-109.

Olson, A.J., Prinja, A.K., Franke, B.C., 2014. Trans. Am. Nucl. Soc. 111, 651.

895 Pomraning, G.C., 1991. Linear kinetic theory and particle transport in stochastic mixtures, World Scientific Publishing, River Edge, NJ, USA.

Pomraning, G.C., 1991. Nucl. Sci. Eng. 108, 325-330.

Pomraning, G.C., 1992. Ann. Nucl. Energy 19, 737-763.

Pomraning, G.C., 1999. Ann. Nucl. Energy 26, 217-235.

900 Santaló, L.A., 1976. Integral geometry and geometric probability, Addison-Wesley, Reading, MA, USA.

Santamarina, A., et al., 2009. The JEFF-3.1.1 Nuclear Data Library, JEFF Report 22, OECDNEA Data Bank.

Schneider, R., Weil, W., 2008. Stochastic and integral geometry, Springer, Berlin, Germany.

905 Schneider, R., 2013. Extremal properties of random mosaics. In: Geometry - Intuitive, Discrete, and Convex. A Tribute to Làszlò Fejes Tòth (I. Bàràny, K. J. Böröczky, G. Fejes Tòth, J. Pach, eds), Bolyai Soc. Math. Studies 24, 301-330, Springer, Berlin.

Schneider, R., 2016. J. Math. Anal. Appl. 434, 1365-1375.

Serra, J., 1982. Image analysis and mathematical morphology, Academic Press, London, UK.

910 Su, B., Pomraning, G.C., 1995. J. Quant. Spectrosc. Radiat. Transfer 54, 779-801.

Tonoike, K., et al., 2013. In: Proceedings of GLOBAL 2013, Salt Lake City, Utah, USA, 729735.

Torquato, S., 2013. Random heterogeneous materials: microstructure and macroscopic properties, Springer-Verlag, New York, USA.

915 Truchet, G., Leconte, P., Santamarina, A., Damian, F., Brun, E., Zoia, A., 2015. Ann. Nuc. Energy 85, 17.

Williams, M.M.R., 1970. Nucl. Sci. Eng. 40, 144-150. 
Williams, M.M.R., 1974. Atomkernenergie 22, 248.

Williams, M.M.R., 2000. Nucl. Sci. Eng. 136, 34-58.

920 Williams, M.M.R., Larsen, E.W., 2001. Nucl. Sci. Eng. 139, 66-77.

Williams, M.M.R., 2003. Nucl. Sci. Eng. 143, 1-18.

Williams, M.M.R., 2004. Ann. Nucl. Energy 31, 1073-1081.

Williams, M.M.R., 2013. Ann. Nucl. Energy 52, 22-27.

Yamamoto, T., 2010. Ann. Nucl. Energy 37, 398-405.

925 Zimmerman, G.B., 1990. Lawrence Livermore National Laboratory Report UCRL-JC-105616.

Zimmerman, G.B., Adams, M.L., 1991. Trans. Am. Nucl. Soc. 66, 287.

Zoia, A., Dumonteil, D., Mazzolo, A., 2012. EPL 100, 40002.

Zuchuat, O., Sanchez, R., Zmijarevic, I., Malvagi, F., 1994. J. Quant. Spectrosc. Radiat. Transfer 51 689-722. 


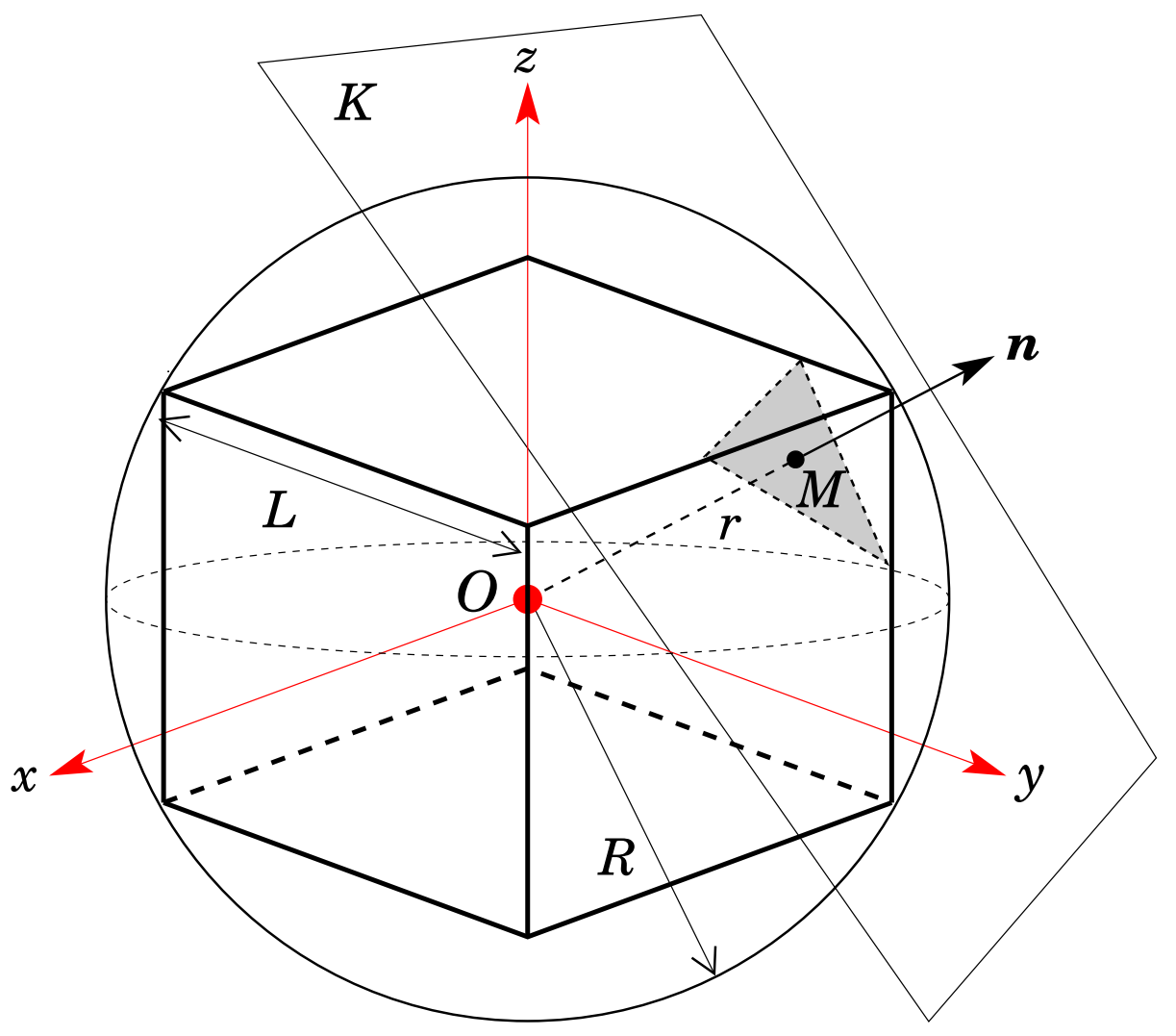

Figure 1: Cutting a cube in $\mathbb{R}^{3}$ with a random plane. A cube of side $L$ is centered in $O$. The circumscribed sphere centered in $O$ has a radius $R=\sqrt{3} L / 2$. The point $\mathbf{M}$ is defined by $\mathbf{M}=r \mathbf{n}$, where $r$ is uniformly sampled in the interval $[-R, R]$ and $\mathbf{n}$ is a random unit vector sampled from $H(\mathbf{n})$. The random plane $K$ of equation $n_{1} x+n_{2} y+n_{3} z=r$ is orthogonal to the vector $\mathbf{n}$ and intersects the point $\mathbf{M}$. The components of $\mathbf{n}$ can be expressed in spherical coordinates as: $n_{1}=\sin (\theta) \cos (\phi), n_{2}=\sin (\theta) \sin (\phi)$, and $n_{3}=\cos (\theta)$, where $\theta$ is the polar angle (projection onto the $z$ axis), and $\phi$ is the azimuthal angle (projection onto the $x-y$ plane). 

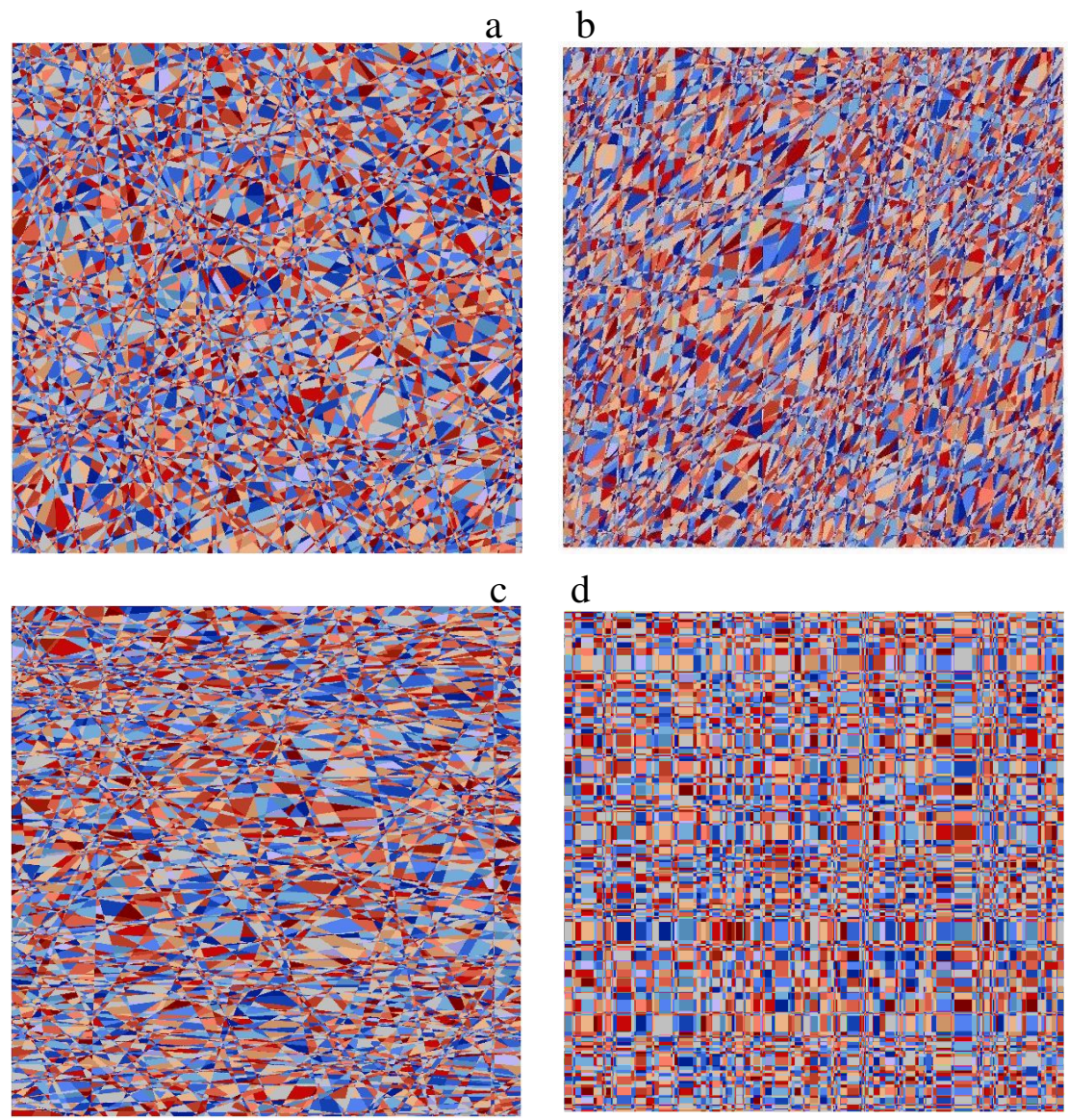

d

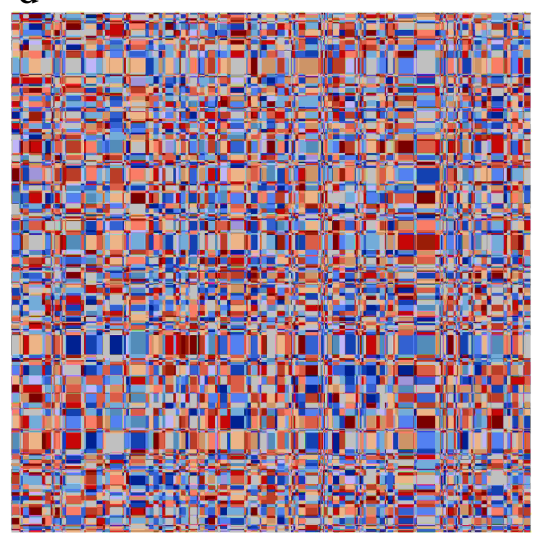

Figure 2: Two-dimensional realizations of anisotropic Poisson tessellations with various angular laws $H(\mathbf{n})=H(\theta), \theta \in[0, \pi]$. For $d=2, \Omega_{2}^{+}=\pi$. The cases illustrated here are as follows: a) isotropic distribution, with $H(\theta)=1 / \pi$; b) quadratic distribution, with $H(\theta)=3 \theta^{2} / \pi^{3}$; c) symmetric histogram distribution, centered at $\theta=\pi / 2$ and having maxima at $\theta=0$ and $\theta=\pi$; d) box distribution, with $H(\theta)=(1 / 2) \delta(\theta)+(1 / 2) \delta(\theta-\pi / 2)$. 

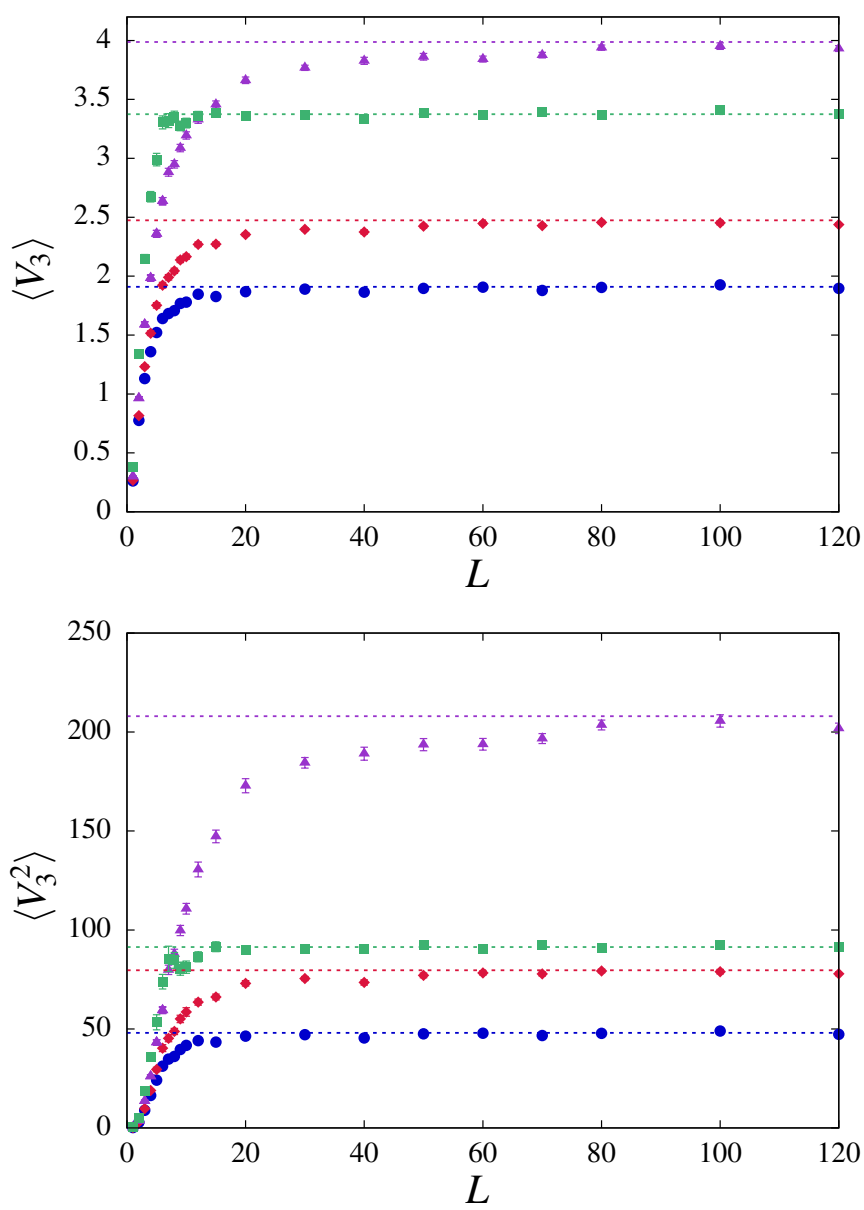

Figure 3: Average volume $\left\langle V_{3}\right\rangle$ (top) and second moment of the volume $\left\langle V_{3}^{2}\right\rangle$ (bottom) of the Poisson tessellation cells as a function of the box side $L$, with various anisotropy laws $H(\mathbf{n})$, for $d=3$. The tessellation density is $\rho=1$ for all the angular laws $H(\mathbf{n})$. Symbols denote Monte Carlo simulation results: blue circles correspond to isotropic distribution, red diamonds to quadratic distribution, green squares to box distribution and purple triangles to histogram distribution. Solid lines correspond to the respective limits for infinite Poisson tessellations, as given in Eqs. (13) and (16), respectively. 

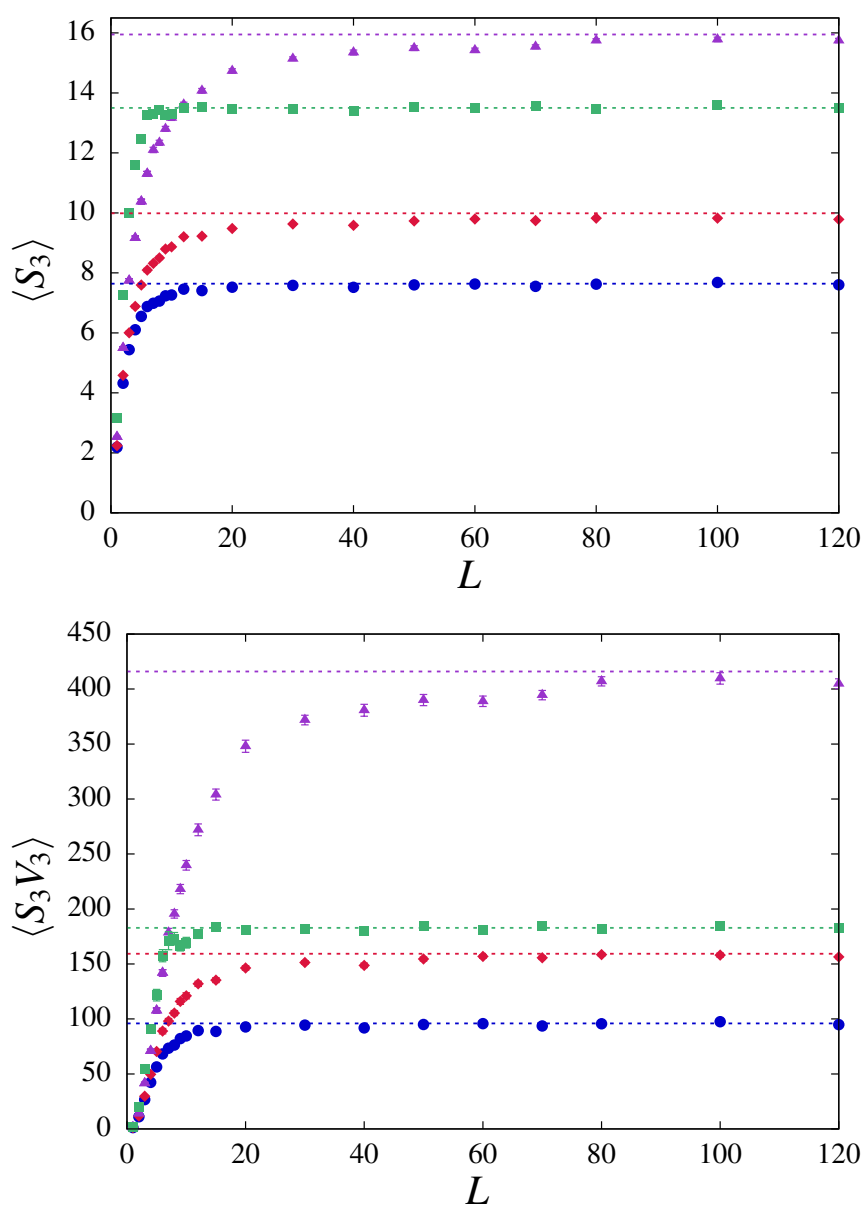

Figure 4: Average surface $\left\langle S_{3}\right\rangle$ (top) and volume-surface correlations $\left\langle V_{3} S_{3}\right\rangle$ (bottom) of the Poisson tessellation cells as a function of the box side $L$, with various anisotropy laws $H(\mathbf{n})$, for $d=3$. The tessellation density is $\rho=1$ for all the angular laws $H(\mathbf{n})$. Symbols denote Monte Carlo simulation results: blue circles correspond to isotropic distribution, red diamonds to quadratic distribution, green squares to box distribution and purple triangles to histogram distribution. Solid lines correspond to the respective limits for infinite Poisson tessellations, as given in Eqs. (10) and (11), respectively. 


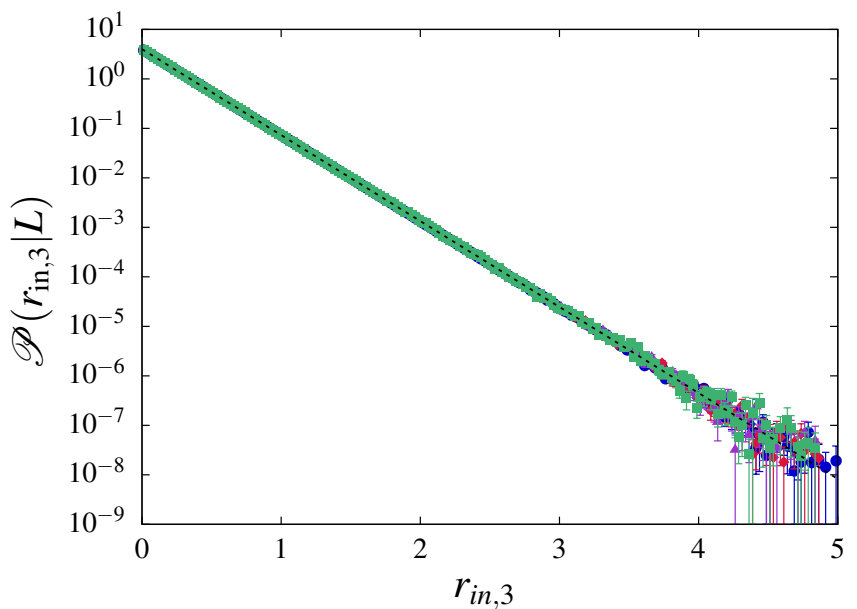

Figure 5: Distribution of the in-radius $r_{\mathrm{in} .3}$ of the Poisson tessellation cells for a box with side $L=120$, with various anisotropy laws $H(\mathbf{n})$, for $d=3$. The tessellation density is $\rho=1$ for all the angular laws $H(\mathbf{n})$. Symbols denote Monte Carlo simulation results: blue circles correspond to isotropic distribution, red diamonds to quadratic distribution, green squares to box distribution and purple triangles to histogram distribution. The solid line corresponds to the limit distribution for infinite Poisson tessellations, i.e., the exponential density given in Eq. (46), independent of $H(\mathbf{n})$. 


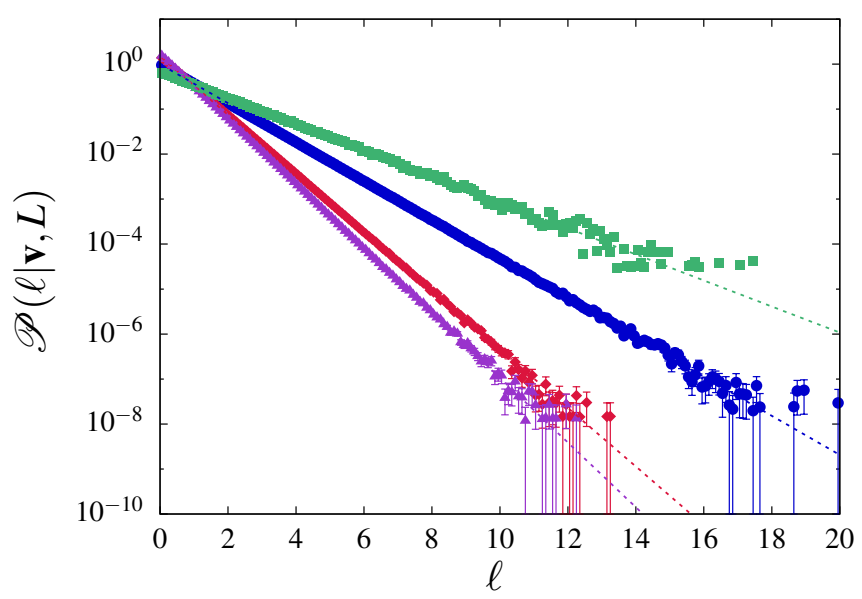

Figure 6: Chord length distribution $\mathscr{P}(\ell \mid \mathbf{v})$ of the Poisson tessellation cells for a box with side $L=120$, with various anisotropy laws $H(\mathbf{n})$, for $d=3$. The tessellation density is $\rho=1$ for all the angular laws $H(\mathbf{n})$. The line orientation is chosen as $\phi=\theta=\pi / 4$. Symbols denote Monte Carlo simulation results: blue circles correspond to isotropic distribution, red diamonds to quadratic distribution, green squares to box distribution and purple triangles to histogram distribution. The solid lines corresponds to the limit distribution for infinite Poisson tessellations, i.e., the exponential density given in Eq. (31), with average $\Lambda(\mathbf{v})$ depending on the orientation $\mathbf{v}$ and on the law $H(\mathbf{n})$ (see Eq. (30)). For the isotropic distribution we have $\Lambda(\mathbf{v})=1$, for the quadratic distribution $\Lambda(\mathbf{v})=2 / 3$, for the box distribution $\Lambda(\mathbf{v})=3 / 2$, and for the histogram distribution $\Lambda(\mathbf{v}) \simeq 0.60383$ 


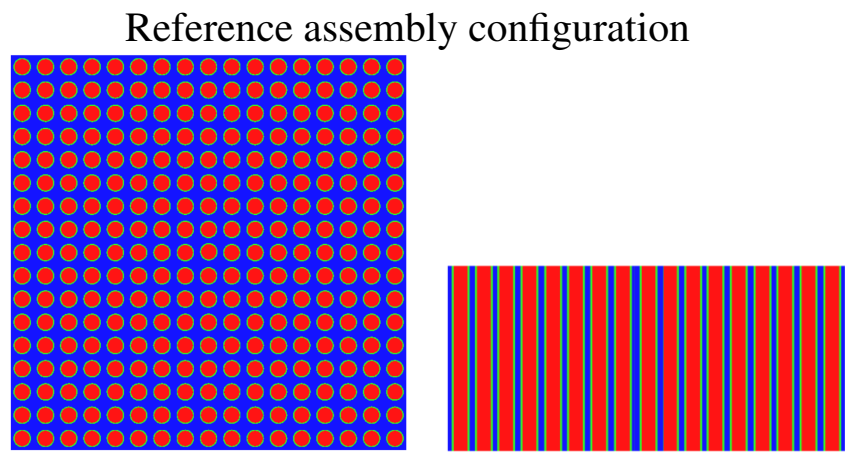

Assembly after random fuel degradation
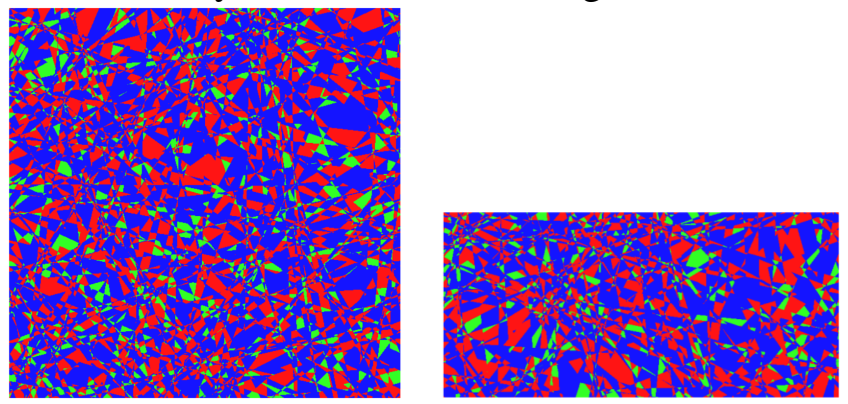

Figure 7: Top. The geometry of the intact $17 \times 17$ fuel assembly that has been chosen for the benchmark. Fuel is displayed in red, cladding in green and moderator in blue. Bottom. Example of fuel degradation obtained by replacing the reference assembly by a realization of an isotropic Poisson tessellation and preserving the average volume ratios for fuel (red), cladding (green) and moderator (blue). Left column: radial view. Right column: axial view.
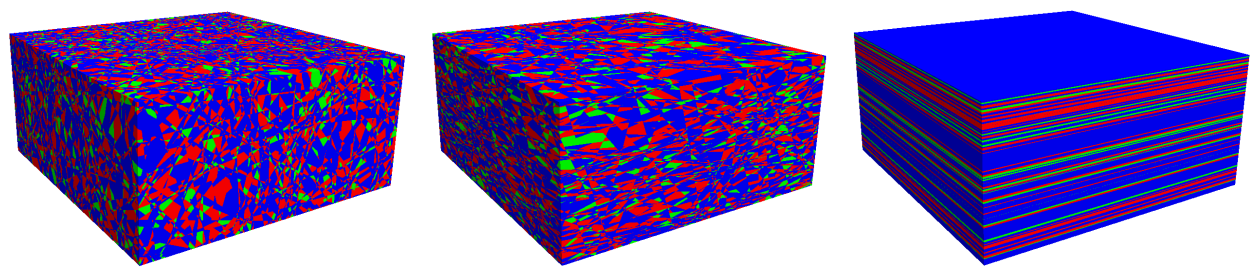

Figure 8: Examples of realizations of three-dimensional anisotropic Poisson tessellations as a model of damaged fuel assembly. Left. Isotropic angular distribution. Center. Histogram angular distribution. Right. Slab angular distribution. The average chord length for the three realizations is $\Lambda=0.15 \mathrm{~cm}$. Red corresponds to fuel, green to cladding and blue to moderator. 

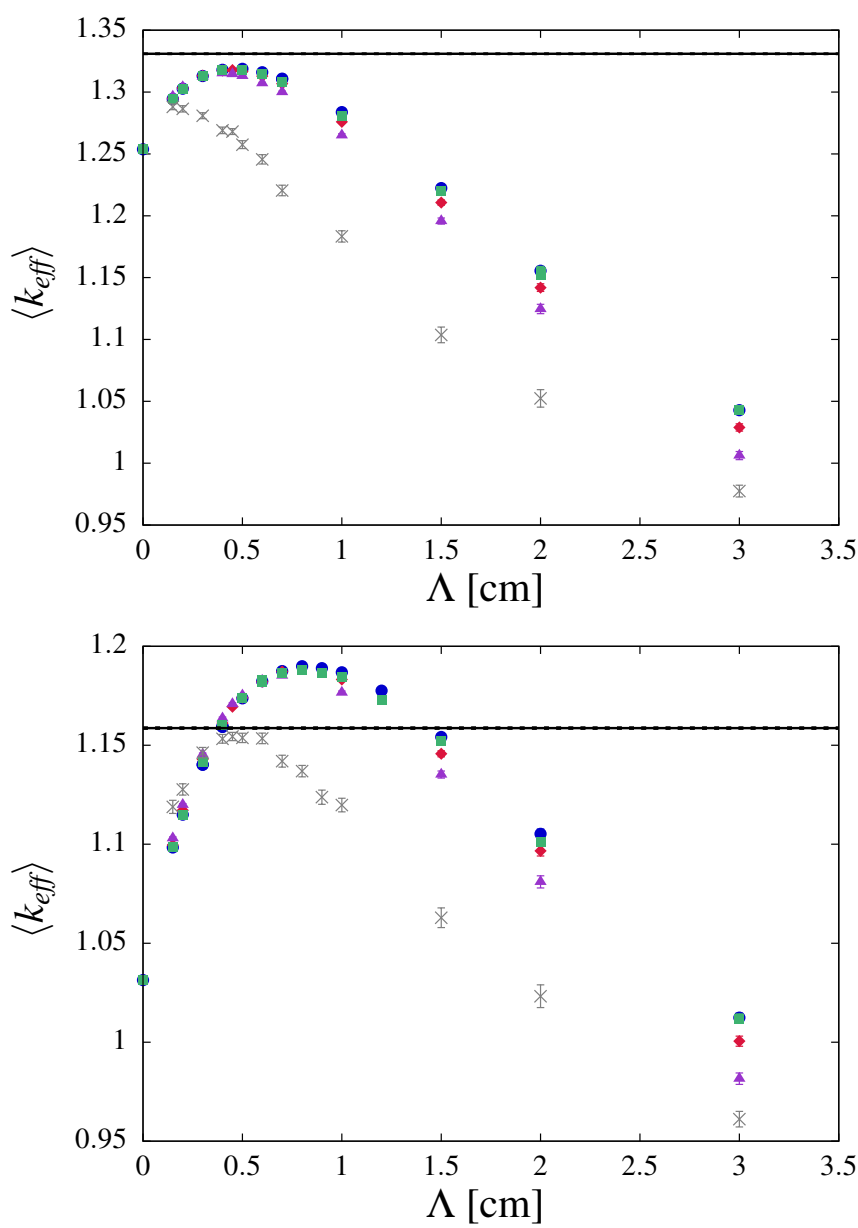

Figure 9: Evolution of the ensemble-averaged multiplication factor $\left\langle k_{\text {eff }}\right\rangle$ as a function of the average chord length $\Lambda$ and of the anisotropy law $H(\mathbf{n})$. Top: UOX assembly. Bottom: MOX assembly. Symbols denote Monte Carlo simulation results: blue circles correspond to isotropic distribution, red diamonds to quadratic distribution, purple triangles to histogram distribution, green squares to box distribution and gray crosses to slab distribution. The limit case at $\Lambda=$ 0 corresponds to the atomic mix model. The black solid line denotes $k_{\text {eff }, 0}$, the result for the assembly with intact fuel pins, which has been added for reference (dashed lines represent the $1 \sigma$ statistical uncertainty). 

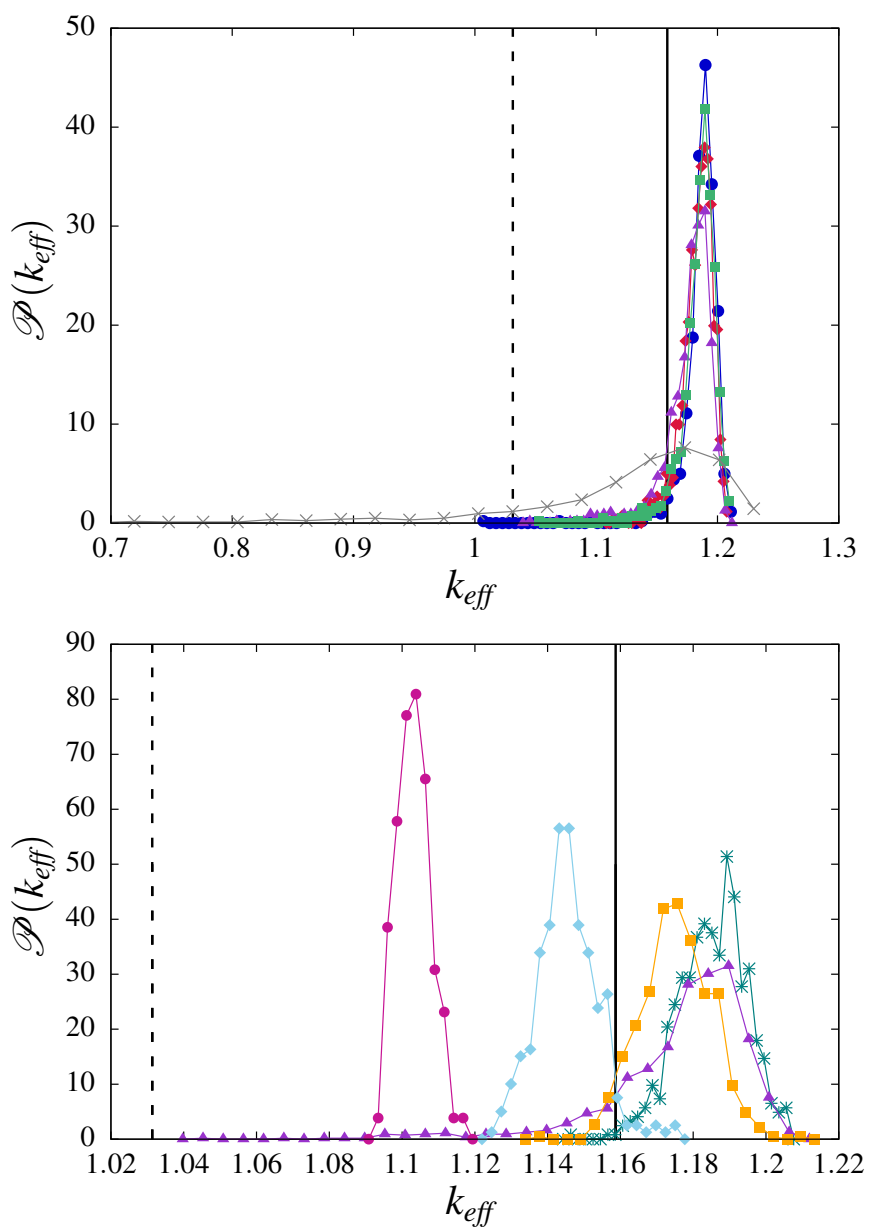

Figure 10: Distributions $\mathscr{P}\left(k_{\text {eff }}\right)$ of the multiplication factor $k_{\text {eff }}$ for MOX configurations. Top. Impact of the anisotropy law $H(\mathbf{n})$ for fixed $\Lambda$. Blue circles correspond to isotropic case, red diamonds to quadratic case, purple triangles to histogram case, green squares to box case and gray crosses to slab case, for $\Lambda=1 \mathrm{~cm}$. Bottom. Impact of the average chord length $\Lambda$ for fixed $H(\mathbf{n})$. Magenta circles represent $\Lambda=0.15 \mathrm{~cm}$, light blue diamonds $\Lambda=0.3 \mathrm{~cm}$, orange squares $\Lambda=0.5 \mathrm{~cm}$, dark green crosses $\Lambda=0.7 \mathrm{~cm}$ and purple triangles $\Lambda=1 \mathrm{~cm}$, for the histogram anisotropy law. The reference values for the case of the intact assembly and for the atomic mix regime are shown as a solid black line and as a dashed black line, respectively. 

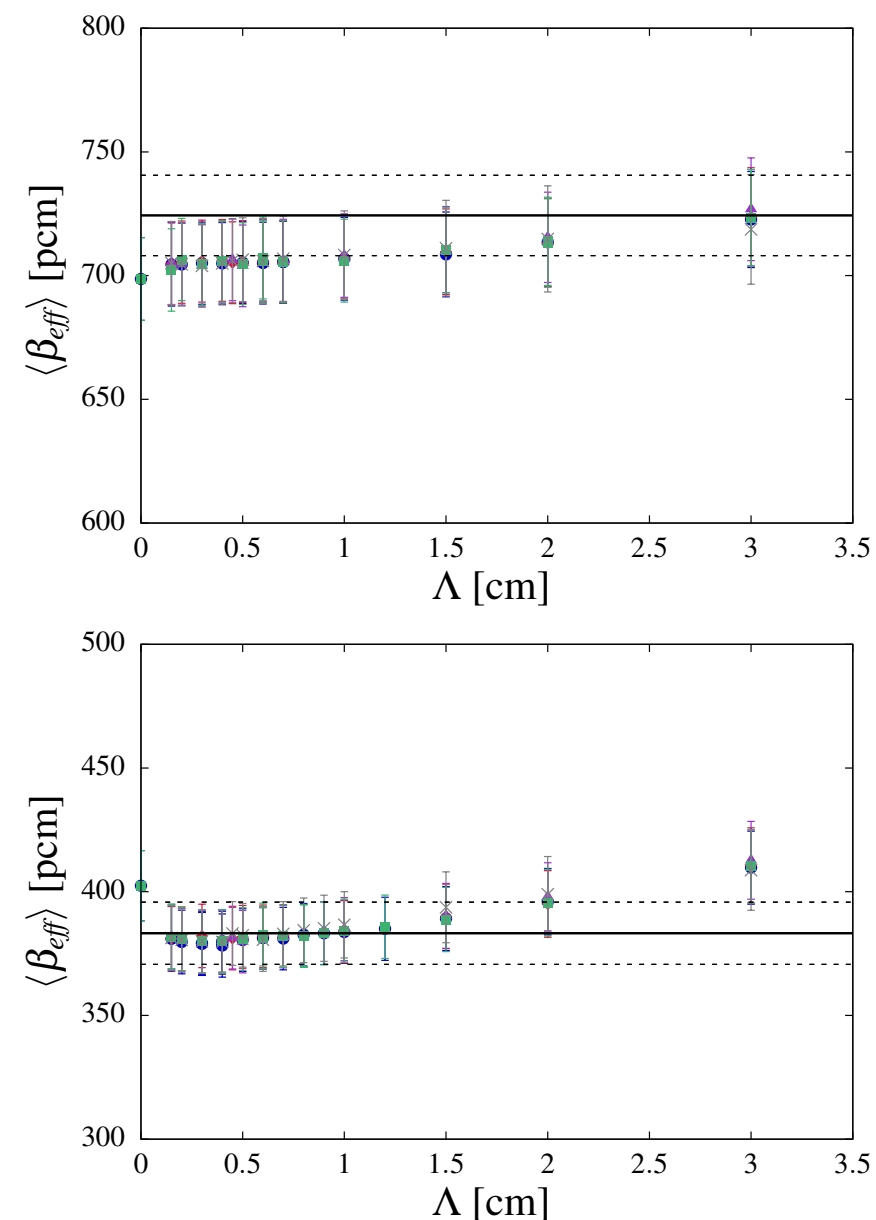

Figure 11: Evolution of the ensemble-averaged effective delayed neutron fraction $\left\langle\beta_{\text {eff }}\right\rangle$ as a function of the average chord length $\Lambda$ and of the anisotropy law $H(\mathbf{n})$. Top: UOX assembly. Bottom: MOX assembly. Symbols denote Monte Carlo simulation results: blue circles correspond to isotropic distribution, red diamonds to quadratic distribution, purple triangles to histogram distribution, green squares to box distribution and gray crosses to slab distribution. The limit case at $\Lambda=0$ corresponds to the atomic mix model. The black solid line denotes $\beta_{\text {eff, }, 0}$, the result for the assembly with intact fuel pins, which has been added for reference (dashed lines represent the $1 \sigma$ statistical uncertainty). 

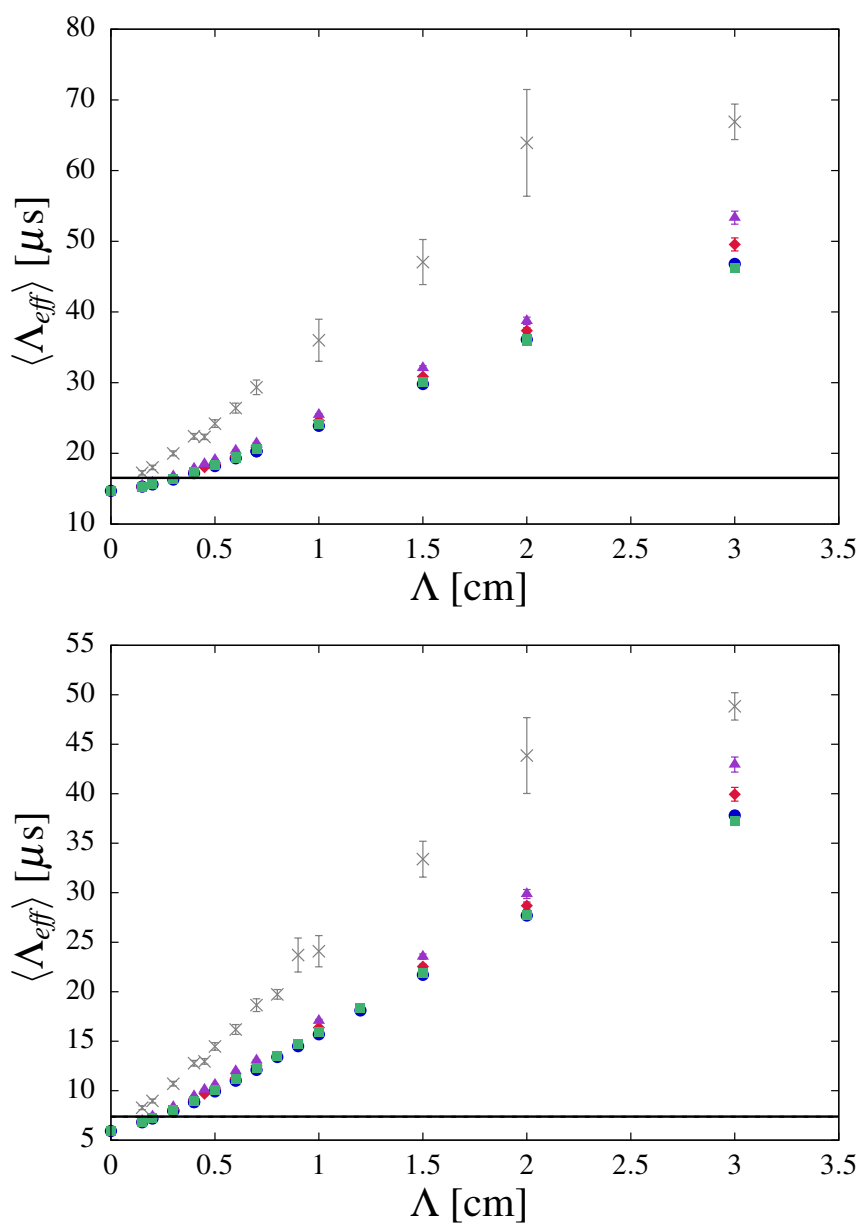

Figure 12: Evolution of the ensemble-averaged effective neutron generation time $\left\langle\Lambda_{\text {eff }}\right\rangle$ as a function of the average chord length $\Lambda$ and of the anisotropy law $H(\mathbf{n})$. Top: UOX assembly. Bottom: MOX assembly. Symbols denote Monte Carlo simulation results: blue circles correspond to isotropic distribution, red diamonds to quadratic distribution, purple triangles to histogram distribution, green squares to box distribution and gray crosses to slab distribution. The limit case at $\Lambda=0$ corresponds to the atomic mix model. The black solid line denotes $\Lambda_{\text {eff, },}$, the result for the UOX assembly with intact fuel pins, which has been added for reference (dashed lines represent the $1 \sigma$ statistical uncertainty). 

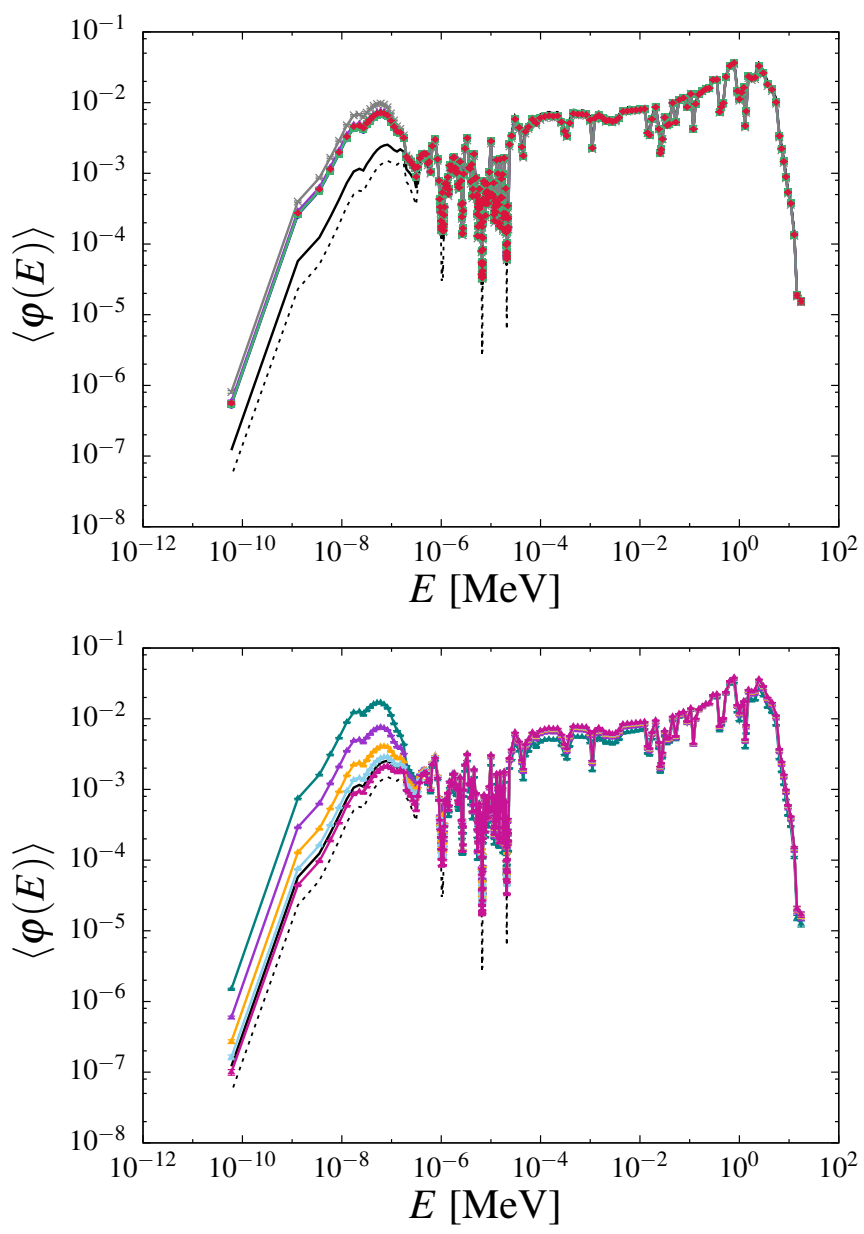

Figure 13: Fundamental eigenmode $\langle\varphi(E)\rangle$ for MOX fuel. Top. Impact of the anisotropy law $H(\mathbf{n})$ for fixed $\Lambda$. Blue circles correspond to isotropic distribution, red diamonds to quadratic distribution, purple triangles to histogram distribution, green squares to box distribution and gray crosses to slab distribution, for $\Lambda=1 \mathrm{~cm}$. Bottom. Impact of the average chord length $\Lambda$ for fixed $H(\mathbf{n})$. Dark green symbols represent results for $\Lambda=3 \mathrm{~cm}$, purple symbols for $\Lambda=1$ $\mathrm{cm}$, orange symbols for $\Lambda=0.5 \mathrm{~cm}$, light blue symbols for $\Lambda=0.3 \mathrm{~cm}$ and magenta symbols for $\Lambda=0.15 \mathrm{~cm}$, for the histogram anisotropy law. The reference spectra for the case of the intact assembly and for the atomic mix regime are shown as a solid black line and as a dashed black curve, respectively. 